\title{
Extended coupled-cluster method. IV. An excitation energy functional and applications to the Lipkin model
}

\author{
N. I. Robinson and R. F. Bishop \\ Department of Mathematics, University of Manchester Institute of Science and Technology, P.O. Box 88, \\ Manchester M60 1QD, England \\ J. Arponen \\ Department of Theoretical Physics, University of Helsinki, Siltavuorenpenger 20C, SF-00170 Helsinki, Finland
}

(Received 10 April 1989)

\begin{abstract}
Earlier papers in this series have shown how the extended coupled-cluster method (ECCM) provides a widely applicable biorthogonal formulation of quantum many-body and quantum field theory. Rules for calculating matrix elements of arbitrary operators have been formulated, and an exact description of the excited states has been given in terms of a generalized random-phase approximation for the many-body, quasilocal c-number (classical), ECCM cluster amplitudes which completely and exactly parametrize the system. The present paper introduces a variational functional in order to treat the excited states differently within the ECCM framework, so as to allow extra flexibility in the choice of approximation schemes. The method is tested on the SU(2) quasispin model of Lipkin, Meshkov, and Glick [Nucl. Phys. 62, 188 (1965); 62, 199 (1965)], which contains the spontaneous breakdown of parity, and which models a shape transition in finite atomic nuclei. The method very well describes both the ground and excited states of the model Hamiltonian over a broad range of coupling parameters on both sides of the critical value corresponding to the transition.
\end{abstract}

\section{INTRODUCTION}

The model many-body Hamiltonian of Lipkin, Meshkov, and Glick ${ }^{1,2}$ (the LMG model) was originally invented to simulate the collective monopole vibrations of spherical atomic nuclei. In view of the complexity of both the most realistic interaction Hamiltonians and the many-body calculations that must employ them, such simple models as that of LMG have played an increasingly important role as a testing ground for new techniques and approximation schemes in many-body theory.

In this respect, although the LMG model is mathematically sufficiently simple to allow a direct numerical solution to essentially arbitrary accuracy, it is nevertheless sophisticated enough to incorporate highly nontrivial physical features. It includes, for example, the possibility of a vibration-induced instability of the nucleus which it models, against a deformation of shape away from sphericity. The model also displays the distinctive features associated with the subtle transition from the limit of a few-body problem, to the thermodynamic limit of an infinite extensive system. As we have already indicated, the LMG model has had an important history as a "homework" test problem against which the more indirect many-body approximation methods have been matched and thereby sharpened. Indeed, the model has proved to be of sufficient challenge for it to have been tested against a large number of theoretical tools. These have comprised both methods which are intrinsically rather universal by nature and which are hence applicable elsewhere, as well as more specialized techniques which are especially adapted to take advantage of the particular mathematical structure of the model. By now the literature on the LMG model is rather extensive, but for a representative selection of some of the more significant works, the interested reader is referred to Refs. 2-10 and to other papers cited therein.

In the present article we are interested in the use of the LMG model to shed further light on what is now regarded as one of the most powerful and most universally applicable of all theoretical frameworks in which to imbed the general quantum many-body problem, namely, the $\exp (S)$ or coupled-cluster method (CCM), as originally invented by Coester ${ }^{11}$ and Coester and Kümmel, ${ }^{12}$ and since widely extended as described further below. In comparison with other techniques of many-body theory, one of the great strengths of the CCM is its combination of very wide generality and great conceptual simplicity. ${ }^{13}$ Since its invention the CCM has been extensively studied and developed. It has by now been widely applied to a diverse array of both realistic and model problems in such fields as nuclear physics, ${ }^{14-19}$ both for finite atomic nuclei and infinite nuclear matter; atomic and molecular systems in quantum chemistry; ${ }^{20-35}$ the homogeneous electron liquid in condensed matter physics; ${ }^{36-39}$ anharmonic oscillators; ${ }^{40-44}$ and relativistic quantum field theory. ${ }^{45-48}$ The CCM has also been rather extensively reviewed in the literature, and the interested reader may be referred in particular to Refs. $13,18,19,26$, and 36 in this regard.

The many-body correlations within the formalism of the CCM enter through the linked-cluster amplitudes which parametrize both the ground state and the excited states, as well as through the specific nonlinear couplings 
of these amplitudes to each other which the method yields. One of the major advantages of the method derives from the fact that it manifestly satisfies the requirement of size extensivity. ${ }^{19,35,49}$ In this respect it may be contrasted with such alternative techniques as the configuration-interaction method, ${ }^{50}$ which contains unlinked diagrams for the energy and hence suffers from the lack of size extensivity. By contrast, the CCM contains no unlinked diagrams for the energy. Accordingly its description of many-body correlation phenomena is in terms of a more localized set of amplitudes. This feature, taken together with the generalized time-ordering properties of the associated generalized tree diagrams, ${ }^{51}$ has proved to be very useful for a description of few-body systems, as well as for extended systems and field theories, for both of which the size-extensivity feature is vital.

The CCM as thus described above actually parametrizes the ket energy eigenstates of the system in terms of a set of linked-cluster amplitudes constructed with respect to some rather arbitrary (but usually noninteracting) model state. For the calculation of the expectation values of operators other than the Hamiltonian, for which the Schrödinger equation may be employed directly, one clearly also needs the bra eigenstates. The most obvious way of constructing such expectation values would simply involve the bra and ket eigenstates being taken as the manifestly Hermitian conjugate states of each other. However, this direct procedure is not without difficulties, as has been described elsewhere. ${ }^{51,52}$ In particular, for all natural truncations which must be used to implement the method in practice, the direct procedure violates the rather basic Feynman-Hellman theorem, which requires the diagrams for the expectation value of an arbitrary operator to be obtained from the corresponding energy diagrams by replacing each Hamiltonian vertex in turn by the particular operator under consideration. ${ }^{53}$

The problems mentioned above have recently been circumvented ${ }^{51}$ by an extension of the CCM which incorporates a parametrization of the bra eigenstates in terms of both the old and a new set of linked-cluster amplitudes in such a way that the bra and ket eigenstates are not manifestly Hermitian conjugate states. To distinguish this later version of the CCM from its earlier counterpart, we henceforth refer to it as the extended coupled-cluster method (ECCM), and rename the original version the normal coupled-cluster method (NCCM). Just as the NCCM treatment of the Hamiltonian is based in practice on a single similarity transform of it, so the ECCM treatment of an arbitrary operator is based on a double similarity transform. The fact that these transformations, which generate the bra and ket eigenstates, are not unitary, leads to the methods being not explicitly Hermitian. The ECCM formulation in particular may be thought of as a biorthogonal representation of the many-body problem.

The present paper is the fourth in a series which is dedicated to the development and application of the ECCM as a powerful extension of the NCCM, which has itself been demonstrated to be a widely applicable and rather universal tool for tackling the many-body problem, as we have indicated above. We have shown in particular in the first paper of the series ${ }^{52}$ how the ECCM provides a very general description for the density matrix of the many-body system, and how it allows dynamic problems to be treated in a way very analogous to that of classical Hamiltonian mechanics. The second paper in the series ${ }^{54}$ extended the ground-state ECCM developed in the first paper to a description of the excited states. We showed in particular how the excited-state ECCM could be regarded as an exact hierarchical generalization of the well-known random-phase approximation of Bohm and Pines. ${ }^{55}$ In the third paper of this series ${ }^{56}$ the ECCM was applied to the zero-temperature hydrodynamics of a strongly interacting condensed Bose fluid. We demonstrated specifically how all of the usual local hydrodynamic balance equations can be formulated microscopically within the ECCM, and that they are obeyed furthermore not only in the full (untruncated) theory, but also at various levels of truncation. The ECCM has also been applied elsewhere ${ }^{57}$ to the problem of a charged impurity in a polarizable medium, which is of importance for the problem of positron annihilation in metals, for example.

The present article treats the excited states within the ECCM framework in an alternative way to that described in the earlier second article of this series ${ }^{54}$ by introducing a new variational functional for them. Just as previously, by use of the dynamical method of small oscillations, ${ }^{51,54}$ so the excitation energies here are also obtained directly by the new method, rather than as the difference between the absolute energies for the excited and ground states obtained from different calculations. In the present method we also have the additional flexibility, however, of choosing independently the truncations (i.e., the particular finite-order approximations to the full equations) for the ground-state and excited-state correlations. This ECCM procedure is thus very much in the spirit of the NCCM treatment of excited states due to Emrich. ${ }^{58}$

Applications have already been made to the LMG model of both this NCCM approach of Emrich ${ }^{7}$ and the earlier ECCM approach using the dynamical theory of small oscillations. ${ }^{9}$ By way of comparison our new approach described here is therefore also applied to the LMG model. We show that it is usually capable of providing a very accurate description of both the low and high excitation energies. This is particularly true of the stable spherical phase. In the highly deformed region the method also works very well, with the exception of not being able to resolve easily the nearly degenerate lowlying parity doublets. The accuracy also diminishes for the strongly fluctuating configurations in the immediate vicinity of the shape (phase) transition, in the subtle intermediate region between a few-body and a real many-body system. These are precisely the regions for which all general methods have had great difficulties. Indeed, most have broken down altogether in one or more of these regions. Therefore, despite the above caveats, we believe that the application of the present method to the LMG model proves it to be of considerable power and generally superior to most alternative techniques of wide applicability. 
The new energy functional for excited states is introduced in Sec. II and then compared with the earlier methods of Ref. 54. This initial discussion is completely general, and hence not restricted to any particular system. The general properties of the LMG model are described in Sec. III, with particular attention paid to the associated spontaneous breaking of the symmetry. In Sec. IV the variationally derived eigenvalue equations are written down in detail, and some exact results are described. The numerical approximation schemes to the exact equations, and the numerical results obtained from using them, are discussed at length in Sec. V. This discussion is divided into two parts, according to whether the model state used as input to the method is symmetric or symmetry breaking. Although the exact ECCM equations are ultimately independent of the model state, their truncated counterparts necessarily display some modelstate dependence, which is described. Finally the results are summarized in Sec. VI, and the salient features of the new scheme and its approximate implementation are highlighted.

\section{THE EXCITATION ENERGY FUNCTIONAL}

The full details of the ECCM description of the ground and excited states of a many-body system have been presented in earlier papers in this series. ${ }^{52,54}$ We first review the general concepts very briefly before proceeding to the new alternative description of the excitation spectrum. In so doing it will become apparent that the new ECCM formulation is closer in spirit to the NCCM description of Emrich, ${ }^{58}$ and the particular connections between the two approaches will be exploited and discussed.

The ECCM has as its basis the following double similarity transformation of the many-body Hamiltonian $H$

$$
H \rightarrow \widehat{H} \equiv e^{S^{\prime \prime}} e^{-S} H e^{S} e^{-S^{\prime \prime}},
$$

where the operators $S$ and $S^{\prime \prime}$ exactly parametrize the ground-state bra and ket energy eigenvectors as

$$
\left\langle\Psi_{0}^{\prime}\right| \equiv\langle\Phi| e^{S^{\prime \prime}} e^{-S}
$$

and

$$
\left|\Psi_{0}\right\rangle \equiv e^{S}|\Phi\rangle=e^{S} e^{-S^{\prime \prime}}|\Phi\rangle,
$$

in terms of some suitable normalized model state $|\Phi\rangle$. The only real requirement on the state $|\Phi\rangle$ is that the algebra of all possible operators in the full many-body Hilbert space be spanned by the two Abelian subalgebras of creation and destruction operators defined with respect to the state $|\Phi\rangle$. The assumption is then that these two algebras and the state $|\Phi\rangle$ are cyclic, i.e., that an arbitrary ket state in the Hilbert space can be constructed as a suitable linear combination of states obtained by letting the elements of the creation operator subalgebra act on the cyclic vector $|\Phi\rangle$, and similarly for the bra states. Thus, in particular, the operators $S$ and $S^{\prime \prime}$ are defined to contain, respectively, only creation parts and only destruction parts with respect to the state $|\Phi\rangle$,

$$
\left\langle\Phi\left|S=0=S^{\prime \prime}\right| \Phi\right\rangle .
$$

The second equality in Eq. (2.4) permits us to write the second equation in Eq. (2.3) as a consequence of the first defining relation.

Furthermore, we may define a complete set of orthonormal creation operators $\left\{C_{i}^{\dagger}\right\}$ with respect to the model or generalized vacuum state $|\Phi\rangle$ by the orthogonality relation

$$
\left\langle\Phi\left|C_{i} C_{j}^{\dagger}\right| \Phi\right\rangle=\delta(i, j),
$$

where $\delta(i, j)$ is a standard Kronecker symbol, and the completeness relation

$$
\begin{aligned}
I & =\sum_{i} C_{i}^{\dagger}|\Phi\rangle\langle\Phi| C_{i} \\
& =|\Phi\rangle\left\langle\Phi\left|+\sum_{i}^{\prime} C_{i}^{\dagger}\right| \Phi\right\rangle\langle\Phi| C_{i},
\end{aligned}
$$

where $I$ is the identity operator and the prime notation on the sum introduced in Eq. (2.6) is defined to exclude from the sum the term $i=0$, which is defined to pick out the identity operator $I \equiv C_{0}^{\dagger}$ from the complete set $\left\{C_{i}^{\dagger}\right\}$. The index $i$ is here clearly some suitable many-body configuration-space index depending on the details of the problem. It is simply a shorthand notation for some suitable set of single-particle indices, each one of which in turn completely specifies a single-particle state in some chosen basis. Examples of the many-body configuration space and the corresponding choices for the indices $\{i\}$ have been given elsewhere. ${ }^{52}$ In terms of these configuration-space creation and destruction operators, the operators $S$ and $S^{\prime \prime}$ have the exact representations

$$
\begin{aligned}
& S \equiv \sum_{i}^{\prime} S_{i} C_{i}^{\dagger}, \\
& S^{\prime \prime} \equiv \sum_{i}^{\prime} S_{i}^{\prime \prime} C_{i} .
\end{aligned}
$$

A key feature of the ECCM is that each of the $c$-number configuration-space amplitudes $\left\{S_{i}, S_{i}^{\prime \prime}\right\}$ represents a linked-cluster amplitude which obeys the cluster property, and which has a well-defined diagrammatic representation. ${ }^{51}$ These properties stem directly from the exponential structure of Eqs. (2.2) and (2.3).

From Eqs. (2.1)-(2.3), the ground-state bra and ket eigenvalue problems

$$
H\left|\Psi_{0}\right\rangle=E_{g}\left|\Psi_{0}\right\rangle, \quad\left\langle\Psi_{0}^{\prime}\right| H=E_{g}\left\langle\Psi_{0}^{\prime}\right|
$$

may thus be written in the equivalent form

$$
\hat{H}|\Phi\rangle=E_{g}|\Phi\rangle, \quad\langle\Phi| \hat{H}=E_{g}\langle\Phi| .
$$

The same defining equations also imply the normalization conditions

$$
\left\langle\Phi \mid \Psi_{0}\right\rangle=\left\langle\Psi_{0}^{\prime} \mid \Psi_{0}\right\rangle=\langle\Phi \mid \Phi\rangle \equiv 1 .
$$

We further note that although $\left\langle\Psi_{0}^{\prime}\right| \propto\left(\left|\Psi_{0}\right\rangle\right)^{\dagger}$ for a Hermitian Hamiltonian $H$, the exact representations of Eqs. (2.2) and (2.3) do not make this condition apparent, since the similarity transformations are certainly not unitary. It is in order to remind ourselves of this lack of manifest Hermiticity that the prime notation is used on the bra eigenstate of Eq. (2.2). The expectation value of the Hamiltonian in its ground state is 


$$
\begin{aligned}
E_{g}=\langle H\rangle & \equiv\left\langle\Psi_{0}^{\prime}|H| \Psi_{0}\right\rangle \\
& =\langle\Phi|\hat{H}| \Phi\rangle \\
& =\left\langle\Phi\left|e^{S^{\prime \prime}} e^{-S} H e^{S}\right| \Phi\right\rangle
\end{aligned}
$$

It is clear that this expectation value may thus be regarded as a functional of the complete set of parameters $\left\{S_{i}, S_{i}^{\prime \prime}\right\}$. Its minimization with respect to each of these parameters is fully equivalent to the Schrödinger equations (2.9), and these coupled nonlinear equations may be solved, in principle, to obtain the complete ECCM parametrization in terms of the operators $S$ and $S^{\prime \prime}$. It has been pointed out elsewhere ${ }^{51,52}$ that a more convenient choice of dual amplitudes is the equivalent set $\left\{\sigma_{i}, \widetilde{\sigma}_{i}\right\}$ defined as

$$
\sigma_{i} \equiv\left\langle\Phi\left|C_{i} e^{S^{\prime \prime}} S\right| \Phi\right\rangle, \quad \widetilde{\sigma}_{i} \equiv S_{i}^{\prime \prime} .
$$

Each member of this new set of amplitudes is also fully linked, and hence obeys the cluster property. The energy expectation value of Eq. (2.12) may also be formally expressed in terms of the set $\left\{\sigma_{i}, \widetilde{\sigma}_{i}\right\}$. Each of the resulting terms again corresponds to a fully connected diagram for the energy, and the Goldstone linked-cluster theorem ${ }^{59}$ is thereby satisfied.

By analogy with the work of Emrich $^{58}$ within the NCCM, a pair of ECCM excitation operators $X^{\lambda}$ and $Y^{\lambda}$ was introduced in the second paper of the present series, ${ }^{54}$ for each of the excited states labeled by the index $\lambda$. The corresponding excited-state bra and ket vectors are defined as

$$
\left\langle\Psi_{\lambda}^{\prime}\right| \equiv\langle\Phi| Y^{\lambda} e^{S^{\prime \prime}} e^{-S}
$$

and

$$
\left|\Psi_{\lambda}\right\rangle \equiv e^{S_{e}-S^{\prime \prime}} X^{\lambda}|\Phi\rangle
$$

The operators $X^{\lambda}$ and $Y^{\lambda}$ are again parametrized in terms of creation pieces only and destruction pieces only, respectively, as

$$
X^{\lambda} \equiv \sum_{i} X_{i}^{\lambda} C_{i}^{\dagger}
$$

and

$$
Y^{\lambda} \equiv \sum_{i} Y_{i}^{\lambda} C_{i}
$$

and where for the exact description of the excited states,

$$
X_{0}^{\lambda}=0=Y_{0}^{\lambda}
$$

since we require $\left\langle\Psi_{\lambda}^{\prime} \mid \Psi_{0}\right\rangle=0=\left\langle\Psi_{0}^{\prime} \mid \Psi_{\lambda}\right\rangle$ for all $\lambda(\neq 0$, by definition). The expectation value of the Hamiltonian in these excited states is given as

$$
E_{g}+\varepsilon_{\lambda} \equiv\left\langle\Psi_{\lambda}^{\prime}|H| \Psi_{\lambda}\right\rangle=\left\langle\Phi\left|Y^{\lambda} \hat{H} X^{\lambda}\right| \Phi\right\rangle,
$$

with $\varepsilon_{\lambda}$ thus defined as the excitation energy. It was shown in the earlier papers ${ }^{52,54}$ how the right-hand side of Eq. (2.19) can be written in terms of the second functional derivatives of $\langle H\rangle$ with respect to the complete set of ECCM amplitudes $\left\{\sigma_{i}, \widetilde{\sigma}_{i}\right\}$ specified by Eq. (2.13). It was further shown ${ }^{54}$ how the excitation amplitudes $\left\{X_{i}^{\lambda}, Y_{i}^{\lambda}\right\}$ and the excitation energy $\varepsilon_{\lambda}$ could be obtained as the solutions to the eigenvalue equations

$$
\sum_{j}^{\prime}\left(\frac{\delta^{2}\langle H\rangle}{\delta \widetilde{\sigma}_{i} \delta \sigma_{j}}+\sum_{k}^{\prime} \sigma_{i+k} \frac{\delta^{2}\langle H\rangle}{\delta \sigma_{k} \delta \sigma_{j}}\right) X_{j}^{\lambda}=\varepsilon_{\lambda} X_{i}^{\lambda},
$$

and

$\sum_{j}^{\prime} Y_{j}^{\lambda}\left(\frac{\delta^{2}\langle H\rangle}{\delta \widetilde{\sigma}_{j} \delta \sigma_{i}}+\sum_{k}^{\prime} \sigma_{j+k} \frac{\delta^{2}\langle H\rangle}{\delta \sigma_{k} \delta \sigma_{i}}\right)=\varepsilon_{\lambda} Y_{i}^{\lambda}$,

where the $\sigma$ amplitude with a compound index is defined as

$$
\sigma_{i+k} \equiv\left\langle\Phi\left|C_{i} C_{k} e^{S^{\prime \prime}} S\right| \Phi\right\rangle
$$

by analogy with Eq. (2.13). It has been explicitly demonstrated $^{54}$ that the excitation spectrum obtained from Eqs. (2.20) and (2.21) is exactly equivalent to that given by considering the effective Hamiltonian that governs the dynamics of small oscillations about the stationary ground state. It was further shown how this latter procedure provides an exact generalization of the wellknown random-phase approximation, and thereby how the ECCM amplitudes $\left\{\sigma_{i}, \widetilde{\sigma}_{i}\right\}$ may be regarded as a complete set of generalized mean many-body fields for the clusters parametrized by the configuration-space indices $\{i\}$.

In order to solve either the ground-state equations

$$
\frac{\delta\langle H\rangle}{\delta \sigma_{i}}=0=\frac{\delta\langle H\rangle}{\delta \widetilde{\sigma}_{i}}
$$

or the excited-state equations (2.20) and (2.21), it is obviously necessary to truncate the equations by some approximation scheme. The most natural way of doing this for the ground state, and that which is employed in this paper, is the so-called $\operatorname{SUB}(n)$ approximation wherein the configuration-space indices $\{i\}$ in either the set $\left\{S_{i}, S_{i}^{\prime \prime}\right\}$ or the set $\left\{\sigma_{i}, \widetilde{\sigma}_{i}\right\}$ of correlation amplitudes are restricted to involve, at most, $n$ particles, and the amplitudes with more than $n$ particles are set to zero. Extending this to the excited states yields the so-called $\operatorname{SUB}(m, n)$ approximation in which the ground-state amplitudes are truncated at $n$ th-order clusters and the excited-state amplitudes $\left\{X_{i}^{\lambda}, Y_{i}^{\lambda}\right\}$ at $m$ th-order clusters, with all higher-order amplitudes put equal to zero.

One of the major features of the NCCM is that all of the hierarchical equations are of finite order in the correlation amplitudes, even for infinite systems. Unfortunately, the double similarity transformation of the ECCM spoils this property. The resulting ECCM equations are of finite order only for problems with a Hilbert space of finite dimension, such as the LMG model, although even here it is the dimension of the space which limits the order of the equations. In order to keep the ECCM equations as compact as possible, and partially to circumvent this problem, it turns out to be more practicable to revert to the parametrization of the ground state in terms of the original amplitudes $\left\{S_{i}, S_{i}^{\prime \prime}\right\}$ rather than the derived dual amplitudes $\left\{\sigma_{i}, \widetilde{\sigma}_{i}\right\}$. We thus employ the ground-state energy functional of Eq. (2.12), and now seek a new comparable functional for the excited states.

To this end we consider the Schrödinger equation for 
an excited ket state in the form

$$
\hat{H} X^{\lambda}|\Phi\rangle=\left(E_{g}+\varepsilon_{\lambda}\right) X^{\lambda}|\Phi\rangle,
$$

in which the amplitudes $\left\{S_{i}, S_{i}^{\prime \prime}\right\}$ inherent in the definition of $\hat{H}$ are assumed to have been determined already by a ground-state ECCM calculation. We now define a new excitation operator $S^{\lambda}$ to be the creation part (with respect to the model state $|\Phi\rangle$ ) of the operator $\exp \left(-S^{\prime \prime}\right) X^{\lambda}$, namely,

$$
S^{\lambda}|\Phi\rangle \equiv e^{-S^{\prime \prime}} X^{\lambda}|\Phi\rangle
$$

with

$$
S^{\lambda}=\sum_{i} S_{i}^{\lambda} C_{i}^{\dagger}
$$

Equation (2.24) may then be written as

$$
e^{S^{\prime \prime}} e^{-S} H e^{S} S^{\lambda}|\Phi\rangle=\left(E_{g}+\varepsilon_{\lambda}\right) e^{S^{\prime \prime}} S^{\lambda}|\Phi\rangle,
$$

and using the fact that the operators $S$ and $S^{\lambda}$ commute, the excited-state equation (2.27) may be combined with its ground-state counterpart of Eq. (2.10) to obtain the result

$$
e^{S^{\prime \prime}} e^{-S}\left[H, S^{\lambda}\right] e^{S}|\Phi\rangle=\varepsilon_{\lambda} e^{S^{\prime \prime}} S^{\lambda}|\Phi\rangle,
$$

in which the ground-state energy $E_{g}$ has been eliminated. Finally, taking the inner product of Eq. (2.28) with the state $\langle\Phi| Y^{\lambda}$ allows us to form the new excited-state functional for the excitation energy,

$$
\varepsilon_{\lambda}\left[S_{i}^{\lambda}, Y_{i}^{\lambda}\right]=\frac{\left\langle\Phi\left|Y^{\lambda} e^{S^{\prime \prime}} e^{-S}\left[H, S^{\lambda}\right] e^{S}\right| \Phi\right\rangle}{\left\langle\Phi\left|Y^{\lambda} e^{S^{\prime \prime}} S^{\lambda}\right| \Phi\right\rangle} .
$$

We note that a comparison of Eqs. (2.13) and (2.25) shows that the relationship between the excited-state amplitudes $S_{i}^{\lambda}$ and $X_{i}^{\lambda}$ is of precisely the same form as that between the ground-state amplitudes $S_{i}$ and $\sigma_{i}$. We thus see rather clearly that the present ECCM treatment of excited states has been formulated in a manner quite analogous to that in which the ground state was originally conceived. ${ }^{51}$

It is clear from the above derivation that the amplitudes $\left\{S_{i}^{\lambda}, Y_{i}^{\lambda}\right\}$, taken in their entirety, completely specify the excited states. If we therefore take the functional derivatives of Eq. (2.29) with respect to each of these amplitudes separately, the stationarity conditions yield the two sets of generalized eigenvalue equations

$$
\left\langle\Phi\left|Y^{\lambda} e^{S^{\prime \prime}} e^{-S}\left[H, C_{i}^{\dagger}\right] e^{S}\right| \Phi\right\rangle-\varepsilon_{\lambda}\left\langle\Phi\left|Y^{\lambda} e^{S^{\prime \prime}} C_{i}^{\dagger}\right| \Phi\right\rangle=0
$$

and

$$
\left\langle\Phi\left|C_{i} e^{S^{\prime \prime}} e^{-S}\left[H, S^{\lambda}\right] e^{S}\right| \Phi\right\rangle-\varepsilon_{\lambda}\left\langle\Phi\left|C_{i} e^{S^{\prime \prime}} S^{\lambda}\right| \Phi\right\rangle=0,
$$

which are fully equivalent to the original bra and ket Schrödinger equations, respectively. We note that Eqs. (2.30) and (2.31) formally run over all values of the index set, including the value $i=0$ which we have used to label the special operator $C_{0}^{\dagger}=I=C_{0}$. Suppose that we now approximate the description of the excited states by a truncation scheme as described above, so that the number of nonzero independent amplitudes $\left\{S_{i}^{\lambda}\right\}$ and $\left\{Y_{i}^{\lambda}\right\}$ is $(M+1)$ each, including the special value $i \rightarrow 0$. The total number of independent excited-state parameters in the truncated scheme is then $(2 M+2)$. However, the functional $\varepsilon_{\lambda}$ of Eq. (2.29) is a homogeneous function of order zero in the coefficients $\left\{S_{i}^{\lambda}, Y_{i}^{\lambda}\right\}$. Multiplication of either set of parameters by an arbitrary constant factor leaves $\varepsilon_{\lambda}$ unchanged, so that the number of independent parameters reduces to $2 M$. An examination of Eq. (2.30) with $i \rightarrow 0$ immediately yields the relation $\varepsilon_{\lambda} Y_{0}^{\lambda}=0$. Since the case of zero excitation energy is presumed to be a solution of the ground-state equation, we must have $Y_{0}^{\lambda}=0$ as remarked previously in Eq. (2.18) for the earlier exact description. Furthermore, since the functional of Eq. (2.29) does not then depend at all on $S_{0}^{\lambda}$, we may also set it to zero with no loss of generality. We are thus left with the $2 M$ effective parameters $\left\{S_{1}^{\lambda}, \ldots, S_{M}^{\lambda}\right\}$ and $\left\{Y_{1}^{\lambda}, \ldots, Y_{M}^{\lambda}\right\}$. Of course the homogeneity of the functional $\varepsilon_{\lambda}$ still implies that only $(2 M-2)$ of these parameters are completely independent, but this is of no concern for the eigenvalue equations as presented in Eqs. (2.30) and (2.31), but where the index set $\{i\}$ now excludes the value $i \rightarrow 0$. The remaining uncertainty in the eigenfunctions is simply related to the normalization of the corresponding excited-state wave functions.

It is also of interest to inquire about the linked-cluster nature of the various operators that have been introduced within the ECCM. In the first place, we stress again that for the ground-state bra and ket eigenvectors, both parametrizations $\left\{S_{i}, S_{i}^{\prime \prime}\right\}$ and $\left\{\sigma_{i}, \widetilde{\sigma}_{i}\right\}$ lead to fully linked terms. Thus while these two sets of cluster amplitudes represent different sets of diagrams for the ground state, it should be noted carefully that both sets represent connected diagrams only. Second, we emphasize that two of the main features of the present approach to the excited states are its variational character and the fact that the method acts in some sense to fully algebraize the manybody problem. In this regard, both the underlying variational formulation of the ECCM approach and the explicit similarity transformations built fundamentally into it, are sufficient to meet most of the demands imposed by considerations of linkedness and separability. Third, we note that the stationary equation (2.30), for example, which determines the excitation amplitudes $\left\{Y_{i}^{\lambda}\right\}$, formally does lead to the apparent appearance of disconnected terms in their solutions. Nevertheless, one may show that when the ground-state cluster amplitudes $\left\{S_{i}, S_{i}^{\prime \prime}\right\}$, which are used as input, are exact, then these disconnected terms explicitly and precisely cancel each other so that only fully linked contributions remain. The precise diagrammatic content and meaning of the present excitedstate ECCM formalism will be analyzed elsewhere.

There has been a considerable amount of discussion lately, at least within the context of applications of CCM (and other) techniques to problems in quantum chemistry, of the related concepts of separability, linkedness, and connectedness of various correlation and cluster operators, and the allied features of size extensivity and size consistency for the formalisms adopting them. This discussion has largely arisen from the various extensions to open-shell systems of the earlier NCCM techniques for 
closed-shell systems. All such attempts are now rooted ultimately in the degenerate versions of perturbation theory. The distinctions and interconnections between the above closely connected concepts become of particular relevance in the latter regard with respect to the formulation of such open-shell techniques in the so-called incomplete model spaces. A further discussion of these matters would take us too far afield for present purposes, but the interested reader is referred to Ref. 60 for a recent review. It is not trivial to discuss precisely how these various properties are exhibited by the present excited-state formulation of the ECCM, since their usual definitions are couched within the language of degenerate perturbation theory, particularly in terms of an effective Hamiltonian in some suitably defined projected model space. Since our own formulation is based rather on the variational functional of Eq. (2.29), we have not attempted a precise translation. Nevertheless, just as Emrich ${ }^{58}$ has explicitly demonstrated within the NCCM both that the corresponding excitation operator is a linked operator and that no disconnected terms appear in its counterpart to Eq. (2.31), so the same is also true for the ECCM equations $[(2.30)$ and $(2.31)]$, at least when the ground-state operators $\left\{S, S^{\prime \prime}\right\}$ are not approximated. It is thus clear that both formulations give expressions for the excitation energies, obtained as eigenvalues, which are size extensive in the sense of scaling properly with particle number $N$ as $N \rightarrow \infty$.

It is worth noting that if in Eq. (2.31) we make the replacement $\exp \left(S^{\prime \prime}\right) \rightarrow I$, this equation simply reduces to the ordinary NCCM excited-state equation of Emrich. ${ }^{58}$ Furthermore, this is precisely analogous to the way in which the ECCM equation for the ground-state ket wave function reduces to its NCCM counterpart of Coester and Kümmel, ${ }^{12}$ thereby reinforcing our earlier remarks about the connections between this new ECCM formulation and the older NCCM.

The other advantage of the functional of Eq. (2.29) for the excitation energies $\varepsilon_{\lambda}$ is its very analogous form to the ground-state functional $E_{g}$ of Eq. (2.12). Indeed, there is an underlying hidden symmetry between the terms in the two expressions involving the similarity transforms with reference to the operator $S$ of the operators $\left[H, S^{\lambda}\right]$ and $H$, respectively. By the usual nested commutator expansion we may write

$$
\begin{aligned}
e^{-S} H e^{S}= & H+[H, S]+\frac{1}{2 !}[[H, S], S] \\
& +\frac{1}{3 !}[[[H, S], S], S]+\cdots .
\end{aligned}
$$

As usual, since $S$ contains only creation operators (with respect to the state $|\Phi\rangle$ ), this otherwise infinite expansion will terminate at a finite order depending on the nature of the Hamiltonian $H$. For example, if $H$ contains products of up to $2 k$ single-particle creation and destruction operators, as would be the case for a system of particles interacting via $k$-body forces, the expansion (2.32) would terminate after the term involving the $2 k$-fold commutator. The comparable term in the numerator of Eq. (2.29) is similarly expanded as

$$
\begin{aligned}
e^{-S}\left[H, S^{\lambda}\right] e^{S}= & {\left[H, S^{\lambda}\right]+\left[\left[H, S^{\lambda}\right], S\right] } \\
& +\frac{1}{2 !}\left[\left[\left[H, S^{\lambda}\right], S\right], S\right]+\cdots,
\end{aligned}
$$

which again terminates after the term involving the $k$ fold commutator. A comparison of Eqs. (2.32) and (2.33) clearly shows that the terms in the numerator of the excitation energy functional $\varepsilon_{\lambda}$ of Eq. (2.29), which involve $S^{\lambda}$ from the expression given in Eq. (2.33), may be found from the comparable terms in the ground-state energy functional $E_{g}$ of Eq. (2.12), which emanate from the expression given in Eq. (2.22), by replacing in each such term every amplitude $S_{i}$ in turn by the corresponding amplitude $S_{i}^{\lambda}$. The terms involving no $S_{i}$ factors in Eq. (2.32) are simply ignored in this procedure. Thus, for example, a term involving the product $S_{i} S_{j}$ in Eq. (2.23) has as its counterpart in Eq. (2.33) the combination $\left(S_{i}^{\lambda} S_{j}+S_{i} S_{j}^{\lambda}\right)$. This procedure has its exact counterpart in the NCCM treatment of excited states of Emrich, ${ }^{58}$ who refers to the corresponding relationship between the ground- and excited-state equations as " $(g, e)$ symmetrization."

This processes can now be completed in our ECCM procedure by comparing the remaining terms in the ground-state functional of Eq. (2.12) and the numerator of the excitation-energy functional of Eq. (2.29). If we denote the operator $\exp \left(S^{\prime \prime}\right)$ by $\Omega$, and its corresponding configuration-space components by $\Omega_{i}$, then it is clear that the remaining part of the corresponding $(g, e)$ symmetrization for our new ECCM approach is the replacement

$$
\Omega_{i} \rightarrow \sum_{j} Y_{j}^{\lambda} \Omega_{i-j},
$$

where the subtracted compound index $(i-j)$ may be defined analogously to the additive compound index of Eq. (2.22), as described more fully elsewhere. ${ }^{52}$ This process, together with the procedure described above, allows us to write down the new ECCM excited-state equations rather easily by these transformations on their corresponding ground-state counterparts.

It is clear that the reformulation of the ECCM for excited states in terms of the amplitudes $\left\{S_{i}^{\lambda}\right\}$ rather than the amplitudes $\left\{X_{i}^{\lambda}\right\}$ has allowed us both to emphasize the links to the NCCM, and to keep the resulting equations as compact and manageable as possible. After this rather general discussion of the method, we turn in Secs. IV and $\mathrm{V}$ to its implementation for the LMG model. The model is first described in Sec. III.

\section{THE LMG MODEL: GENERAL RESULTS AND PRELIMINARIES}

The LMG model consists of $N$ identical fermions distributed between two energy levels, each of which is $N$ fold degenerate. The energy difference between the two levels is scaled to be unity, and the Hamiltonian is then taken as

$$
\begin{aligned}
H= & \frac{1}{2} \sum_{p, m} m a_{p, m}^{\dagger} a_{p, m} \\
& +\frac{1}{2} V \sum_{p, p^{\prime}, m} a_{p, m}^{\dagger} a_{p^{\prime}, m}^{\dagger} a_{p^{\prime},-m} a_{p,-m},
\end{aligned}
$$


where the quantum number $p=1,2, \ldots, N$ labels the degenerate single-particle states within each level, the index $m= \pm 1$ specifies the level in question, and $a_{p, m}^{\dagger}$ and $a_{p, m}$ are the fermion creation and destruction operators, respectively, for a particle in the state $(p, m)$. They obey the usual fermion anticommutation relations. The twobody matrix elements are simply all set equal to the same constant value $\frac{1}{2} V$, and the Hamiltonian has the form of a simple pairing model.

As is well known, the Hamiltonian of Eq. (3.1) may be mapped into a spin-algebraic model by introducing the quasispin operators

$$
\begin{aligned}
& J_{+} \equiv \sum_{p} a_{p,+1}^{\dagger} a_{p,-1} \equiv J_{x}+i J_{y}, \\
& J_{-} \equiv \sum_{p} a_{p,-1}^{\dagger} a_{p,+1} \equiv J_{x}-i J_{y}, \\
& J_{z} \equiv \frac{1}{2} \sum_{p, m} m a_{p, m}^{\dagger} a_{p, m} .
\end{aligned}
$$

It is readily checked from the fermion anticommutation relations that these quasispin operators satisfy the usual angular momentum commutation relations of $\mathrm{SU}(2)$. The Hamiltonian of Eq. (3.1) is then trivially rewritten as

$$
H=J_{z}+\frac{g}{2 N}\left(J_{+}^{2}+J_{-}^{2}\right)
$$

where $g \equiv N V$ is a dimensionless coupling constant. We also easily see that the Hamiltonian conserves the total quasispin

$$
\left[H, J^{2}\right]=0 ; \quad J^{2} \equiv J_{x}^{2}+J_{y}^{2}+J_{z}^{2},
$$

so that the eigenstates of $H$ may be classified into multiplets according to the quantum number $j$ associated with the eigenvalues $j(j+1)$ of the operator $J^{2}$, as usual. The subspace containing the ground state belongs to the multiplet with maximum value of $j$, namely $N / 2$.

It is also clear that the eigenfunctions of the $\mathrm{LMG}$ Hamiltonian having an even (odd) number of particles excited into the upper level may be thought of as having even (odd) parity. The Hamiltonian only affects pairs of particles at a time and hence does not mix states of opposite parity. If we define the parity operator $\Pi$ as

$$
\mathbf{I I} \equiv \exp \left[i \pi\left(J_{z}+j\right)\right]
$$

we then have the result

$$
[H, \mathrm{II}]=0 \text {. }
$$

Finally, we see that the Hamiltonian of Eq. (3.1) has an interaction of monopole-monopole type which scatters particles between the upper and lower levels only if they have the same value of $p$. Thus, if we define the operators $n_{p}$ as

$$
n_{p} \equiv \sum_{m} a_{p, m}^{\dagger} a_{p, m}
$$

we see that the LMG Hamiltonian not only commutes with the operators $J^{2}$ and II, but also with these new operators

$$
\left[H, n_{p}\right]=0 .
$$

For a given number of particles $N$ and interaction strength $g$, the LMG Hamiltonian may then be exactly diagonalized in principle in the usual angular momentum basis, and by taking these additional symmetries into account.

In order to investigate the LMG model with CCM techniques we first need to make some choice of model state $|\Phi\rangle$. An obvious choice is the Slater determinant with all of the particles in the lower level

$$
|\Phi\rangle \equiv \prod_{p=1}^{N} a_{p,-1}^{\dagger}|0\rangle,
$$

where $|0\rangle$ is the vacuum state, $a_{p, m}|0\rangle=0$. This state also has the property that

$$
J_{-}|\Phi\rangle=0 \text {. }
$$

Although, in principle, if we carry out an exact CCM calculation, the choice of model state is irrelevant, in an approximate (or truncated) implementation of the method this is no longer so. In this case, we may wonder whether a better choice of model state is possible. There is no precise way to answer this question without going into the details of the approximation scheme. Nevertheless, a particularly physically appealing argument is to seek that model state $\left|\Phi^{\prime}\right\rangle$ which maximizes the overlap $M$ with the exact wave function $|\Psi\rangle$,

$$
M \equiv \frac{\left|\left\langle\Phi^{\prime} \mid \Psi\right\rangle\right|^{2}}{\left\langle\Phi^{\prime} \mid \Phi^{\prime}\right\rangle\langle\Psi \mid \Psi\rangle}
$$

This maximum overlap condition has been particularly investigated by Kümmel, ${ }^{6}$ who has demonstrated its usefulness for investigating shape transitions of the sort exhibited by the LMG model. In its lowest order the maximization of the overlap $M$ is equivalent to doing a Hartree-Fock calculation to choose the optimal singleparticle basis. The technique is based on the Thouless theorem, ${ }^{53}$ which asserts that any Slater determinant $\left|\Phi^{\prime}\right\rangle$ in the neighborhood of $|\Phi\rangle$, and hence not orthogonal to it, may be written in the form

$$
\left|\Phi^{\prime}\right\rangle=c e^{T}|\Phi\rangle \text {, }
$$

where, apart from some normalization constant $c, T$ is some general one-body operator, i.e., an operator bilinear in the single-particle operators of the creation variety with respect to $|\Phi\rangle$.

For the LMG model Hamiltonian of Eq. (3.1) or (3.5), an explicit form for the operator $T$ is clearly

$$
T=i \gamma J_{+},
$$

where $\gamma$ is a constant. We may further show that $\left|\Phi^{\prime}\right\rangle$ may be written in the same Slater determinantal form as $|\Phi\rangle$

$$
\left|\Phi^{\prime}\right\rangle=\prod_{p=1}^{N} b_{p,-1}^{\dagger}|0\rangle,
$$

in terms of some new set of single-particle fermion operators $b_{p,-1}^{\dagger}$ given by

$$
b_{p,-1}^{\dagger}=\cos \left(\frac{1}{2} \alpha\right) a_{p,-1}^{\dagger}+i \sin \left(\frac{1}{2} \alpha\right) a_{p,+1}^{\dagger},
$$


where $\gamma \equiv \tan \left(\frac{1}{2} \alpha\right)$. Imposition of the fermion anticommutation relations then also implies that the remaining single-particle operators in the transformed basis may be written as

$$
b_{p,+1}^{\dagger}=i \sin \left(\frac{1}{2} \alpha\right) a_{p,-1}^{\dagger}+\cos \left(\frac{1}{2} \alpha\right) a_{p,+1}^{\dagger} .
$$

We may also define a new set of quasispin operators $K_{+}$, $K_{-}$, and $K_{z}$ defined in terms of the transformed operators of Eqs. (3.17) and (3.18) in just the same way as $J_{+}$, $J_{-}$, and $J_{z}$ are defined in terms of the original fermion operators by Eqs. (3.2)-(3.4). We then have the relation

$$
K_{-}\left|\Phi^{\prime}\right\rangle=0
$$

which is the analogue of Eq. (3.12), and the explicit relations

$$
\begin{aligned}
& K_{+}=\cos ^{2}\left(\frac{1}{2} \alpha\right) J_{+}+\sin ^{2}\left(\frac{1}{2} \alpha\right) J_{-}-i(\sin \alpha) J_{z}, \\
& K_{-}=\sin ^{2}\left(\frac{1}{2} \alpha\right) J_{+}+\cos ^{2}\left(\frac{1}{2} \alpha\right) J_{-}+i(\sin \alpha) J_{z}, \\
& K_{z}=-\frac{1}{2} i(\sin \alpha)\left(J_{+}-J_{-}\right)+(\cos \alpha) J_{z} .
\end{aligned}
$$

It is an easy matter to show that Eqs. (3.20a)-(3.20c) may be written in the equivalent form as

$$
\begin{aligned}
K_{x} & =J_{x}, \\
K_{y} & =J_{y} \cos \alpha-J_{z} \sin \alpha \\
& =\exp \left(i \alpha J_{x}\right) J_{y} \exp \left(-i \alpha J_{x}\right), \\
K_{z} & =J_{y} \sin \alpha+J_{z} \cos \alpha \\
& =\exp \left(i \alpha J_{x}\right) J_{z} \exp \left(-i \alpha J_{x}\right) .
\end{aligned}
$$

We therefore also see from Eqs. (3.12) and (3.19) that Eq. (3.14) may be expressed as

$$
\left|\Phi^{\prime}\right\rangle=\exp \left(i \alpha J_{x}\right)|\Phi\rangle .
$$

This is precisely the form of the deformed Hartree-Fock solution that was guessed by Agassi et al., ${ }^{2}$ and it clearly corresponds to the situation in which each of the individual one-particle quasispins, which are all aligned along the $z$ axis for the state $|\Phi\rangle$, is now rotated by the same angle $\alpha$. These authors argued for this state $\left|\Phi^{\prime}\right\rangle$ on symmetry grounds. They simply chose as trial variational wave functions this one-parameter set of states in which all the quasispins are oriented at the angle $\alpha$ with respect to the $z$ axis in the $y z$ plane. They reasoned first that the trial wave function should keep as much symmetry as possible, arguing that only parity should no longer be a good quantum number, but that the deformed state $\left|\Phi^{\prime}\right\rangle$ should still be an eigenfunction of $J^{2}$ and the operators $n_{p}$ of Eq. (3.9). This is analogous to the retention of axial symmetry in the case of deformed nuclei in which spherical symmetry is broken. They showed first that these requirements correspond to a rotation in quasispin space. They further argued that if the total quasispin is rotated, keeping $J^{2}$ and $\left\langle J_{z}\right\rangle$ fixed, then the expectation value of the Hamiltonian of Eq. (3.5) is clearly minimized when $\left\langle J_{x}^{2}\right\rangle$ is minimized and $\left\langle J_{y}^{2}\right\rangle$ is maximized. This clearly corresponds to the case where the quasispin lies in the $y z$ plane.
The Hamiltonian of Eq. (3.5) may be expressed in terms of the new quasispin operators as

$$
\begin{aligned}
& H=(\cos \alpha) K_{z}+\frac{1}{2} i(\sin \alpha)\left(K_{+}-K_{-}\right) \\
& +\frac{g}{2 N}\left[\frac{1}{2}\left(1+\cos ^{2} \alpha\right)\left(K_{+}^{2}+K_{-}^{2}\right)\right. \\
& \quad+\sin ^{2} \alpha\left(K_{+} K_{-}-K_{z}-2 K_{z}^{2}\right) \\
& \left.\quad+i \sin \alpha \cos \alpha\left(2 K_{+} K_{z}-2 K_{z} K_{-}+K_{+}-K_{-}\right)\right],
\end{aligned}
$$

Thus a Hartree-Fock calculation on this Hamiltonian is equivalent to minimizing its expectation value in the state $\left|\Phi^{\prime}\right\rangle$ with respect to the angle $\alpha$. Using Eq. (3.19), and the simple relation that $\left|\Phi^{\prime}\right\rangle$ is an eigenfunction of $K_{z}$ with eigenvalue $(-N / 2)$, we easily find that

$$
\left\langle\Phi^{\prime}|H| \Phi^{\prime}\right\rangle=-\frac{N}{2}\left[\cos \alpha+\frac{g}{2}\left(\frac{N-1}{N}\right) \sin ^{2} \alpha\right] \text {. }
$$

The stationary points of Eq. (3.24) are clearly given by

$$
\sin \alpha=0 \text { or } \cos \alpha=\frac{N}{(N-1) g} .
$$

It is trivial to verify that for $g<N /(N-1)$, the only solutions are $\alpha=0$, which is a minimum, and $\alpha=\pi$, which is a maximum. In this case $(\alpha=0)$ the state $\left|\Phi^{\prime}\right\rangle$ reverts to the original state $|\Phi\rangle$, and this solution is simply the Hartree-Fock approximation for the so-called normal phase, which preserves the parity symmetry of the Hamiltonian.

Conversely, both solutions $\alpha=0, \pi$ are local maxima for the case $g>N /(N-1)$, while the solutions $\alpha= \pm \cos ^{-1}\left[g^{-1} N /(N-1)\right]$ now give two degenerate minima. This case is the Hartree-Fock approximation to the deformed or symmetry-breaking phase, where the eigenstates no longer have parity as a good quantum number. The symmetric case is rather analogous to the real-space one-body problem of a single-well anharmonic oscillator potential, whereas the latter corresponds to the comparable double-well problem. An exact solution of the LMG model shows similar behavior at some critical value of the coupling constant $g_{c} \sim N /(N-1)$. However, for finite values of $N$ the transition is clearly not sharp, since the two otherwise degenerate symmetry-broken parity doublets can communicate via quantum-mechanical tunneling through the finite central barrier. ${ }^{1,2,61}$ Agassi et $a l .{ }^{2}$ have considered this tunneling between the two degenerate Hartree-Fock solutions by projecting out states of good parity in the usual fashion by taking the obvious two equally weighted linear combinations of the two $\left|\Phi^{\prime}\right\rangle$ states. For present purposes, however, we shall apply the CCM techniques directly to either the symmetric state $|\Phi\rangle$ or to one or other of the two symmetrybreaking states $\left|\Phi^{\prime}\right\rangle$, with the respective choices of the angle $\alpha$ as in Eq. (3.25), as described explicitly in Sec. IV.

\section{EXACT RESULTS}

The exact $N$-body ground-state bra and ket wave functions may be readily parametrized for the LMG model in 
terms of the "normal" model state $|\Phi\rangle$ of Eq. (3.11) and the ECCM correlation operators $S$ and $S^{\prime \prime}$ of Eqs. (2.2) and (2.3). In turn, these operators now have the rather natural decompositions

$$
S=N \sum_{n=1}^{N}(i N)^{-n} S_{n} J_{+}^{n}
$$

and

$$
S^{\prime \prime}=N \sum_{n=1}^{N}(-i N)^{-n} S_{n}^{\prime \prime} J_{-}^{n},
$$

in terms of the set of real $c$-number amplitudes $\left\{S_{n}, S_{n}^{\prime \prime} ; n=1, \ldots, N\right\}$. Equations (4.1) and (4.2) are thus the special forms for the LMG model of the more general equations (2.7) and (2.8). The parity eigenstates of the normal phase are parametrized by amplitudes $\left\{S_{n}, S_{n}^{\prime \prime}\right\}$ for which all such coefficients with $n$ odd are identically zero. However, in order to obtain approximate results within the deformed phase we also allow these odd amplitudes to be nonzero in order to break the parity symmetry. It has already been shown ${ }^{9}$ that the ECCM procedure, described more fully below, produces excellent results for the ground-state energy in both phases of the LMG model, with the above parametrization.

A somewhat lengthy but straightforward calculation first gives the expression

$$
e^{-S} H e^{S}|\Phi\rangle=N \sum_{n=0}^{\infty}(i N)^{-n} h_{n} J_{+}^{n}|\Phi\rangle,
$$

where the coefficients $h_{n}$ are given by

$$
h_{n}=-\frac{1}{2} \delta_{n, 0}+n S_{n}-\frac{1}{2} g\left(\delta_{n, 2}+A_{n}\right)
$$

with

$$
\begin{aligned}
A_{n}= & \frac{(N-n)(N-n-1)}{N^{3}}(n+1)(n+2) S_{n+2}+\sum_{j, k} \delta_{j+k, n+2} \frac{j k}{N^{2}}\left[N^{2}-(3 n+1) N+2 n^{2}+2 n+1-j k\right] S_{j} S_{k} \\
& -\frac{2}{N}(N-n) \sum_{j, k, l} \delta_{j+k+l, n+2} j k l S_{j} S_{k} S_{l}+\sum_{j, k, l, m} \delta_{j+k+l+m, n+2} j k l m S_{j} S_{k} S_{l} S_{m} .
\end{aligned}
$$

The operator $\exp \left(S^{\prime \prime}\right)$ may also be written in the form

$$
e^{S^{\prime \prime}} \equiv \sum_{n=0}^{\infty} i^{n} \Omega_{n} J_{-}^{n},
$$

where the coefficients $\Omega_{n}$ may be obtained as multinomials of the correlation amplitudes $\left\{S_{n}^{\prime \prime}\right\}$ by comparison with Eq. (4.2). They are easily obtained recursively as

$$
\begin{aligned}
& \Omega_{0}=1, \\
& \Omega_{n}=\sum_{m=1}^{n} \frac{m}{n} N^{1-m} S_{m}^{\prime \prime} \Omega_{n-m}, \quad n>0 .
\end{aligned}
$$

Finally, using Eqs. (4.3) and (4.6), we can easily express the ground-state energy as a function of the parameters $\left\{S_{n}, S_{n}^{\prime \prime}\right\}$ from Eq. (2.12), in the form

$$
E_{g}=N \sum_{n=0}^{N} K_{n} \Omega_{n} h_{n},
$$

where

$$
K_{n} \equiv \frac{N ! n !}{N^{n}(N-n) !} .
$$

By making use of the $(g, e)$ symmetrization procedure discussed in Sec. III, it is a simple matter to write down the corresponding expression for the excitation-energy functional of Eq. (2.29). If we parametrize the excitation operators $S^{\lambda}$ and $Y^{\lambda}$ in an analogous fashion to the operators $S$ and $S^{\prime \prime}$ above,

$$
\begin{aligned}
& S^{\lambda}=N \sum_{n=1}^{N}(i N)^{-n} S_{n}^{\lambda} J_{+}^{n}, \\
& Y^{\lambda}=N \sum_{n=1}^{N}(-i N)^{-n} Y_{n}^{\lambda} J_{-}^{n},
\end{aligned}
$$

in terms of the $c$-number coefficients $\left\{S_{n}^{\lambda}, Y_{n}^{\lambda}\right\}$, the explicit form of the schematic replacement indicated in Eq. (3.24) becomes

$$
\Omega_{n} \rightarrow \sum_{m=1}^{n} N^{1-m} Y_{m}^{\lambda} \Omega_{n-m} .
$$

The excitation-energy functional is thus readily found as

$$
\varepsilon_{\lambda}=\frac{\sum_{n=1}^{N} \sum_{m=1}^{n} N^{-m} K_{n} Y_{m}^{\lambda} \Omega_{n-m} h_{n}^{\lambda}}{\sum_{n=1}^{N} \sum_{m=1}^{n} N^{-m} K_{n} Y_{m}^{\lambda} \Omega_{n-m} S_{n}^{\lambda}},
$$

where

$$
\begin{aligned}
h_{n}^{\lambda}=n S_{n}^{\lambda}-\frac{1}{2} g & \frac{(N-n)(N-n-1)}{N^{3}}(n+1)(n+2) S_{n+2}^{\lambda} \\
& +2 \sum_{j, k} \delta_{j+k, n+2} \frac{j k}{N^{2}}\left[N^{2}-(3 n+1) N+2 n^{2}+2 n+1-j k\right] S_{j}^{\lambda} S_{k} \\
& \left.-\frac{6}{N}(N-n) \sum_{j, k, l} \delta_{j+k}+l, n+2 j k l S_{j}^{\lambda} S_{k} S_{l}+4 \sum_{j, k, l, m} \delta_{j+k+l+m, n+2} j k \operatorname{lm} S_{j}^{\lambda} S_{k} S_{l} S_{m}\right) .
\end{aligned}
$$


The stationarity condition for $\varepsilon_{\lambda}$ with respect to each of the coefficients $\left\{S_{k}^{\lambda}, Y_{k}^{\lambda}\right\}$, then leads to the two sets of generalized eigenvalue equations,

$$
\begin{aligned}
\sum_{n=1}^{N} \sum_{m=1}^{n} & N^{-m} K_{n} Y_{m}^{\lambda} \Omega_{n-m} \frac{\partial h_{n}^{\lambda}}{\partial S_{k}^{\lambda}} \\
& \quad-\varepsilon_{\lambda} \sum_{m=1}^{k} N^{-m} K_{k} Y_{m}^{\lambda} \Omega_{k-m}=0, \quad k=1,2, \ldots, N,
\end{aligned}
$$

for the amplitudes $\left\{Y_{n}^{\lambda}\right\}$ and

$$
\begin{gathered}
\sum_{n=k}^{N} N^{-k} K_{n} \Omega_{n-k} h_{n}^{\lambda}-\varepsilon_{\lambda} \sum_{n=k}^{N} N^{-k} K_{n} \Omega_{n-k} S_{n}^{\lambda}=0, \\
k=1,2, \ldots, N,
\end{gathered}
$$

for the amplitudes $\left\{S_{n}^{\lambda}\right\}$. We note that the two sets of generalized eigenvalue equations decouple from each oth- er. Each set should produce exactly the same eigenvalue spectrum. In each case the ground-state coefficients $\left\{S_{n}, S_{n}^{\prime \prime}\right\}$ are required as input. They are found by solving the $2 N$ coupled algebraic equations obtained from the stationarity conditions

$$
\partial E_{g} / \partial S_{n}=0=\partial E_{g} / \partial S_{n}^{\prime \prime}, \quad n=1,2, \ldots, N
$$

for the ground-state energy functional $E_{g}$ of Eq. (4.8).

An equivalent analysis also applies if we use the symmetry-broken state $\left|\Phi^{\prime}\right\rangle$, with the Hartree-Fock value $\alpha=\cos ^{-1}\left[g^{-1} N /(N-1)\right]$ as our model state. In this case the Hamiltonian is given by Eq. (3.23), and our parametrization of the operators $S, S^{\prime \prime}, S^{\lambda}$, and $Y^{\lambda}$ is exactly as before except that the operators $K_{+}$and $K_{-}$replace $J_{+}$and $J_{-}$, respectively, in Eqs. (4.1), (4.2), (4.10), and (4.11), and similarly in Eqs. (4.3) and (4.6). The formulation then proceeds exactly as before, except that in the ground-state case the coefficients $h_{n}$ of Eqs. (4.3) and (4.4) are replaced by new coefficients $h_{n}^{\prime}$ given by

$$
\begin{aligned}
& h_{n}^{\prime}= \cos \alpha\left(-\frac{1}{2} \delta_{n, 0}+n S_{n}\right)+\frac{1}{2} \sin \alpha\left(-\delta_{n, 1}-\frac{(N-n)}{N}(n+1) S_{n+1}+\sum_{k, l} \delta_{k+l, n+1} k l S_{k} S_{l}\right) \\
&-\frac{1}{2} g\left[\frac{1}{2}\left(1+\cos ^{2} \alpha\right)\left(\delta_{n, 2}+A_{n}\right)+\frac{\sin ^{2} \alpha}{N}\left(\frac{1}{2}(N-1) \delta_{n, 0}-3(N-n) n S_{n}+3 N \sum_{k, l} \delta_{k+l, n} k l S_{k} S_{l}\right)\right. \\
&+\frac{\sin \alpha \cos \alpha}{N}\left[-(N-1) \delta_{n, 1}+2 N(n-1) S_{n-1}-\frac{1}{N}\left[N^{2}-(3 n+1) N+2 n^{2}+n\right](n+1) S_{n+1}\right. \\
&\left.\left.+3(N-n) \sum_{k, l} \delta_{k+l, n+1} k l S_{k} S_{l}-2 N \sum_{k, l, m} \delta_{k+l+m, n+1} k l m S_{k} S_{l} S_{m}\right)\right]
\end{aligned}
$$

with the counterpart of the excited-state equation (4.14) being deduced from Eq. (4.18) by the same $(g, e)$ symmetrization procedure as for the symmetric Hamiltonian.

It is clear that neither the ground-state nor excitedstate ECCM equations can be solved analytically for arbitrary $N$. Our method of solution will be based on the socalled $\operatorname{SUB}(m, n)$ scheme in which the excitation amplitudes $\left\{S_{i}^{\lambda}, Y_{i}^{\lambda}\right\}$ with $i=m+1, \ldots, N$, and the groundstate correlation amplitudes $\left\{S_{j}, S_{j}^{\prime \prime}\right\}$ with $j=n$ $+1, \ldots, N$ are set to zero, and the remaining truncated equations are solved numerically. If we are interested only in the ground state, the corresponding approximation is denoted as $\operatorname{SUB}(n)$. It is clear that a $\operatorname{SUB}(N)$ or $\operatorname{SUB}(N, N)$ calculation is exact for this system.

We remark that it is possible to give some analytical results in the limit $N \rightarrow \infty$. For example, Arponen and Rantakivi ${ }^{9}$ have derived ground-state ECCM results in the SUB(2) approximation based on the symmetric model state $|\Phi\rangle$ for both the normal and deformed phases. Using these ground-state results as input to our excited-state formalism, it is easy to show that in $\operatorname{SUB}(1,2)$ approximation, for example, the (first) excitation energy is given by

$$
\varepsilon_{1} \underset{N \rightarrow \infty}{\longrightarrow}\left\{\begin{array}{l}
\left(1-g^{2}\right)^{1 / 2}, g<1 \\
{\left[2\left(g^{2}-1\right)\right]^{1 / 2}, g>1}
\end{array}\right.
$$

which is precisely the result obtained from the timedependent Hartree-Fock theory, which is itself exact in this limit $N \rightarrow \infty$.

Before we proceed to our approximate results, we first give some exact results in Table I of $\operatorname{SUB}(N, N)$ calculations for the few-body examples $N=2,4$, and 6 . For such exact calculations the results must be independent of the choice of model state. We have checked that our results are the same for the two choices of normal and deformed Hartree-Fock states, and that they agree with those obtained by a direct exact diagonalization of the Hamiltonian in an angular momentum basis. Further, an examination of the ground-state correlation amplitudes, as shown in Fig. 1 for a typical example, reveals that there is no sharp phase transition at any value of the coupling constant $g$, as expected.

\section{NUMERICAL RESULTS}

We now present results obtained numerically and based on various $\operatorname{SUB}(m, n)$ approximations at several values of the particle number $N$ and over a range of values of the coupling constant that spans the transition from symmetric to deformed ground state in each case. Calculations have been performed using both the "normal" symmetric model noninteracting state $|\Phi\rangle$ of Eq. 
TABLE I. The exact results from full $\operatorname{SUB}(N, N)$ calculations of the few-body problems $N=2,4,6$, for the ground-state correlation energy $E_{0} \equiv-E_{g}-\frac{1}{2} N$ and the excitation energies $\varepsilon_{n}, n=1, \ldots, N$, at various values of the coupling constant $g$.

\begin{tabular}{rccccccr}
\hline \hline$N$ & $g$ & $E_{0}$ & $\varepsilon_{1}$ & $\varepsilon_{2}$ & $\varepsilon_{3}$ & $\varepsilon_{4}$ & $\varepsilon_{5}$ \\
\hline 2 & 0.4 & 0.01980 & 1.01980 & 2.03961 & & & \\
& 1.0 & 0.11803 & 1.11803 & 2.23607 & & & \\
& 2.0 & 0.41421 & 1.41421 & 2.82843 & & & \\
& 5.0 & 1.69258 & 2.69258 & 5.38517 & & & \\
& 0.4 & 0.02978 & 0.98575 & 2.02978 & 3.07381 & 4.05956 & \\
& 1.0 & 0.17945 & 0.92945 & 2.17945 & 3.42945 & 4.35890 & \\
& 2.0 & 0.64575 & 0.84298 & 2.64575 & 4.44853 & 5.29150 & \\
& 5.0 & 2.76970 & 0.88865 & 4.76970 & 8.65074 & 9.53940 & \\
& 0.4 & 0.03344 & 0.96785 & 1.98865 & 3.03344 & 4.07823 & 5.09903 \\
& 1.0 & 0.21149 & 0.83101 & 1.97873 & 3.21149 & 4.44425 & 5.59196 \\
& 2.0 & 0.84406 & 0.57807 & 2.24513 & 3.84406 & 5.44298 & 7.11004 \\
& 5.0 & 4.08960 & 0.33189 & 4.72933 & 7.08960 & 9.44988 & 13.84731 \\
\hline \hline
\end{tabular}

(3.11) and the "deformed" symmetry-breaking HartreeFock model state $\left|\Phi^{\prime}\right\rangle$ of Eqs. (3.16) and (3.17), with $\alpha=\cos ^{-1}\left[g^{-1} N /(N-1)\right]$. In each case the corresponding ground-state ECCM equations (4.17) are first solved in the $\operatorname{SUB}(n)$ approximation. We note that these equations are a coupled set of $2 n$ nonlinear multinomial equations in the parameters $\left\{S_{i}, S_{i}^{\prime \prime} ; i=1, \ldots, n\right\}$. The possibility thus exists for a variety of stationary solutions for the ground-state energy functional $E_{g}$ at each level of approximation. In most cases a single solution is easily found, although others are certainly present. We have not made a systematic search of all such solutions since for present purposes we are more interested in using the ground-state results as input to our linear generalized eigenvalue problem for the excited states. Thus, having specified the ground-state parameters, the excitation amplitudes $\left\{S_{i}^{\lambda}, Y_{i}^{\lambda} ; i=1, \ldots, m\right\}$ are uniquely found in the $\operatorname{SUB}(m, n)$ approximation by solving either of the two sets of linear generalized eigenvalue equations (4.15) or

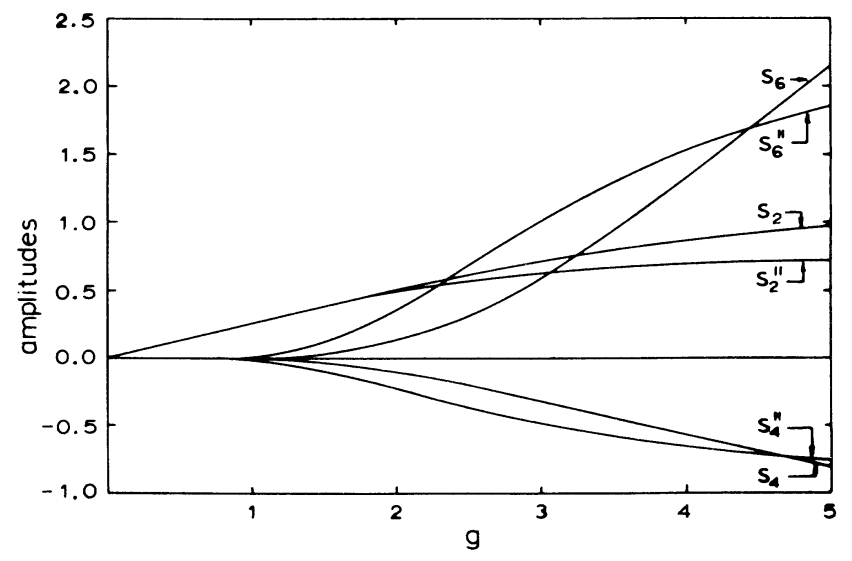

FIG. 1. The exact ground-state correlation coefficients $\left\{S_{i}, S_{i}^{\prime \prime}\right\}$ based on the normal model state, for an $N=6$ system as a function of the coupling parameter $g$. The amplitudes with odd index $i$ are identically zero.
(4.16), where in each case the index $i$ runs over the values $1, \ldots, m$ only. In either case one obtains an approximation to $m$ excitation energies $\varepsilon_{\lambda} \rightarrow\left\{\varepsilon_{1}, \ldots, \varepsilon_{m}\right\}$.

The multiplicity of ground-state approximate solutions has been further discussed by Arponen and Rantakivi, ${ }^{9}$ who at various low levels of approximation investigated several stationary solutions in the vicinity of the true ground-state configuration. In these cases they were able to show by use of a dynamical variational principle for the action, and by linearizing the resulting equations of motion around each stationary point, that many of these additional ground-state stationary solutions were dynamically unstable (i.e., that the corresponding dynamical matrix no longer had purely real eigenvalues). We also briefly touch below in our new ECCM formulation on comparable features of the multiple ground-state solutions. Finally, we note that the interested reader may also consult Refs. 43 and 44 for a fuller discussion of the nonuniqueness of the ground-state solutions for the corresponding NCCM treatment of an anharmonic oscillator.

\section{A. The normal (symmetric) model state}

The ground state of the LMG model using the ECCM based on the normal model state $|\Phi\rangle$ has been extensively studied by Arponen and Rantakivi. ${ }^{9}$ They have demonstrated that even at relatively low values of the $\operatorname{SUB}(n)$ truncation index $n$, the method gives excellent results for the ground-state energy of the system in both phases of the system, so long as the critical point in the coupling constant $g$ is not approached too closely. At a given truncation level, a critical value of $g$ is determined by the point above which a branch of the solutions opens up in which the odd-indexed parameters $\left\{S_{i}, S_{i}^{\prime \prime}\right\}$ become nonzero. Even near this critical value the method is still capable of giving well-behaved results of reasonable accuracy.

We have used the ground-state $\operatorname{SUB}(n)$ results obtained in this way as input for our excited-state equations (4.15)-(4.16), for various values of $N$, and for values of $g$ 
TABLE II. The excitation energies $\varepsilon_{n}$ for the $N=14$ system with $g=0.8$, in various $\operatorname{SUB}(m, n)$ approximations based on the normal (symmetric) model state and labeled by the truncation indices $(m, n)$, compared with the exact results.

\begin{tabular}{lccccccccccccc}
\hline \hline & $(3,4)$ & $(4,4)$ & $(5,4)$ & $(6,4)$ & $(7,4)$ & $(8,4)$ & $(9,4)$ & $(10,4)$ & $(11,4)$ & $(12,4)$ & $(13,4)$ & $(14,4)$ & Exact \\
\hline$\varepsilon_{1}$ & 0.770 & 0.770 & 0.770 & 0.770 & 0.770 & 0.770 & 0.770 & 0.770 & 0.770 & 0.770 & 0.770 & 0.770 & 0.770 \\
$\varepsilon_{2}$ & 1.706 & 1.690 & 1.690 & 1.689 & 1.689 & 1.689 & 1.689 & 1.689 & 1.689 & 1.689 & 1.689 & 1.689 & 1.689 \\
$\varepsilon_{3}$ & 2.735 & 2.735 & 2.700 & 2.700 & 2.696 & 2.696 & 2.696 & 2.696 & 2.696 & 2.696 & 2.696 & 2.696 & 2.696 \\
$\varepsilon_{4}$ & & 3.827 & 3.827 & 3.772 & 3.772 & 3.766 & 3.766 & 3.765 & 3.765 & 3.765 & 3.765 & 3.765 & 3.765 \\
$\varepsilon_{5}$ & & & 4.957 & 4.957 & 4.885 & 4.885 & 4.877 & 4.877 & 4.876 & 4.876 & 4.876 & 4.876 & 4.876 \\
$\varepsilon_{6}$ & & & & 6.102 & 6.102 & 6.023 & 6.023 & 6.014 & 6.014 & 6.014 & 6.014 & 6.014 & 6.013 \\
$\varepsilon_{7}$ & & & & & 7.247 & 7.247 & 7.172 & 7.172 & 7.164 & 7.164 & 7.164 & 7.164 & 7.163 \\
$\varepsilon_{8}$ & & & & & & 8.380 & 8.380 & 8.319 & 8.319 & 8.314 & 8.314 & 8.314 & 8.313 \\
$\varepsilon_{9}$ & & & & & & 9.493 & 9.493 & 9.453 & 9.453 & 9.451 & 9.451 & 9.450 \\
$\varepsilon_{10}$ & & & & & & & 10.581 & 10.581 & 10.562 & 10.562 & 10.561 & 10.561 \\
$\varepsilon_{11}$ & & & & & & & & 11.634 & 11.634 & 11.629 & 11.629 & 11.630 \\
$\varepsilon_{12}$ & & & & & & & & & 12.636 & 12.636 & 12.636 & 12.638 \\
$\varepsilon_{13}$ & & & & & & & & & & 13.555 & 13.555 & 13.556 \\
$\varepsilon_{14}$ & & & & & & & & & & & & & \\
\hline \hline
\end{tabular}

in the range from $0-5$. We have demonstrated explicitly that in each case the truncated equations (4.15) and (4.16) yield identical eigenspectra. Typical results are shown in Tables II and III for the case $N=14$ in various $\operatorname{SUB}(m, n)$ approximation schemes, and for values of $g$ in the normal, symmetry-preserving phase. Comparison is made with exact results obtained from a direct diagonalization of the Hamiltonian in an angular momentum basis. One observes that the results are remarkably accurate, even for ground-state input obtained at a rather low level of approximation. We emphasize that the current method for treating the excited states does not automatically guarantee that the excitation spectrum will become more accurate as the excitation truncation index $m$ is increased towards its maximum value $N$, if the ground-state truncation index is held fixed at some value $n<N$. Nevertheless, our numerical results for the normal phase indicate the very accurate and rapidly converging nature of the results. A similar pattern of accuracy for the various $\operatorname{SUB}(m, n)$ truncations is also exhibited for higher values of the particle number $N$ over the normal phase regime of values of the coupling constant. Typical results for higher $N$ are shown in Table IV. The fact that for the values of $g$ considered in Tables II-IV, each of the states is a parity eigenstate, is reflected in the entries to these tables. Thus, one observes that in proceeding from a $\operatorname{SUB}(m, n)$ to a $\operatorname{SUB}(m+1, n)$ approximation, the computed value for the $i$ th excitation energy $\varepsilon_{i}$ only changes when $(m+1)$ is a multiple of two greater than $i$. This is simply due to the fact that the odd-indexed (evenindexed) excited-state amplitudes $\left\{S_{i}^{\lambda}, Y_{i}^{\lambda}\right\}$ are identically zero for each of the even (odd) excited states, respectively, both for the exact calculation and at the various levels of truncation shown.

In Table $\mathrm{V}$ we also show results using the normal model state for the starting point of a calculation for the deformed phase of the $N=14$ system. We notice in this case the appearance of some eigenvalues which are negative, and others which arise as complex conjugate pairs. It is clear that the ECCM formalism that we are using is not manifestly Hermitian, and hence there is no reason to expect only real eigenvalues at any level of approxima-

TABLE III. The excitation energies $\varepsilon_{n}$ for the $N=14$ system with $g=1.0$, in various $\mathrm{SUB}(m, n)$ approximations based on the normal (symmetric) model state and labeled by the truncation indices $(m, n)$, compared with the exact results.

\begin{tabular}{|c|c|c|c|c|c|c|c|c|c|c|c|c|c|}
\hline & $(3,6)$ & $(4,6)$ & $(5,6)$ & $(6,6)$ & $(7,6)$ & $(8,6)$ & $(9,6)$ & $(10,6)$ & $(11,6)$ & $(12,6)$ & $(13,6)$ & $(14,6)$ & Exact \\
\hline$\varepsilon_{1}$ & 0.651 & 0.651 & 0.649 & 0.649 & 0.648 & 0.648 & 0.648 & 0.648 & 0.648 & 0.648 & 0.648 & 0.648 & 0.648 \\
\hline$\varepsilon_{2}$ & 1.628 & 1.569 & 1.569 & 1.557 & 1.557 & 1.554 & 1.554 & 1.553 & 1.553 & 1.553 & 1.553 & 1.553 & 1.553 \\
\hline$\varepsilon_{3}$ & 2.738 & 2.738 & 2.619 & 2.619 & 2.587 & 2.587 & 2.579 & 2.579 & 2.578 & 2.578 & 2.578 & 2.578 & 2.578 \\
\hline$\varepsilon_{4}$ & & 3.936 & 3.936 & 3.759 & 3.759 & 3.702 & 3.702 & 3.688 & 3.688 & 3.687 & 3.687 & 3.687 & 3.686 \\
\hline$\varepsilon_{5}$ & & & 5.169 & 5.169 & 4.948 & 4.948 & 4.869 & 4.869 & 4.853 & 4.853 & 4.852 & 4.852 & 4.852 \\
\hline$\varepsilon_{6}$ & & & & 6.398 & 6.398 & 6.153 & 6.153 & 6.066 & 6.066 & 6.053 & 6.053 & 6.053 & 6.052 \\
\hline$\varepsilon_{7}$ & & & & & 7.599 & 7.599 & 7.355 & 7.355 & 7.277 & 7.277 & 7.271 & 7.271 & 7.270 \\
\hline$\varepsilon_{8}$ & & & & & & 8.756 & 8.756 & 8.543 & 8.543 & 8.490 & 8.490 & 8.489 & 8.488 \\
\hline$\varepsilon_{9}$ & & & & & & & 9.868 & 9.868 & 9.714 & 9.714 & 9.689 & 9.689 & 9.688 \\
\hline$\varepsilon_{10}$ & & & & & & & & 10.941 & 10.941 & 10.860 & 10.860 & 10.854 & 10.853 \\
\hline$\varepsilon_{11}$ & & & & & & & & & 11.982 & 11.982 & 11.961 & 11.961 & 11.962 \\
\hline$\varepsilon_{12}$ & & & & & & & & & & 12.984 & 12.984 & 12.984 & 12.987 \\
\hline$\varepsilon_{13}$ & & & & & & & & & & & 13.889 & 13.889 & 13.892 \\
\hline$\varepsilon_{14}$ & & & & & & & & & & & & 14.539 & 14.540 \\
\hline
\end{tabular}




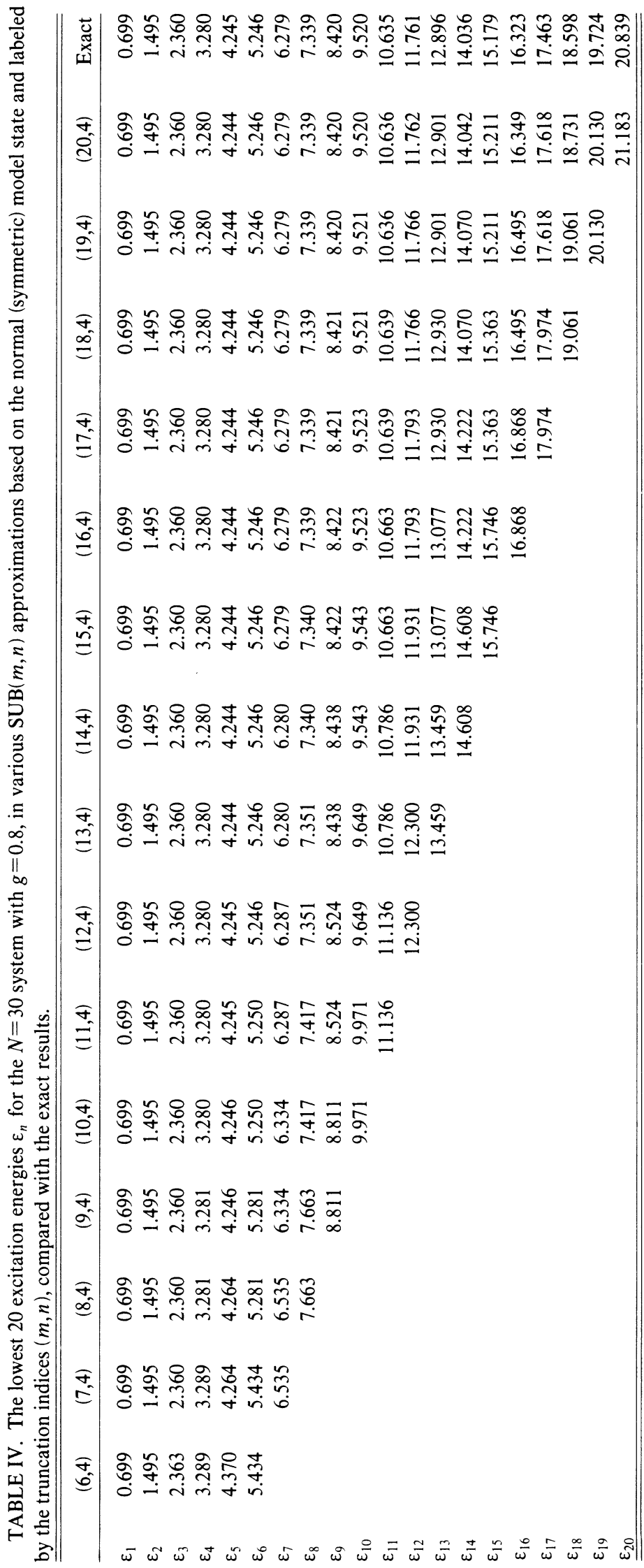


TABLE V. The excitation energies $\varepsilon_{n}$ for the $N=14$ system with $g=5.0$, in various $\operatorname{SUB}(m, n)$ approximations based on the normal (symmetric) model state and labeled by the truncation indices $(m, n)$, compared with the exact results.

\begin{tabular}{|c|c|c|c|c|c|c|c|c|c|c|c|c|c|}
\hline & $(3,4)$ & $(4,4)$ & $(5,4)$ & $(6,4)$ & $(7,4)$ & $(8,4)$ & $(9,4)$ & $(10,4)$ & $(11,4)$ & $(12,4)$ & $(13,4)$ & $(14,4)$ & Exact \\
\hline$\varepsilon_{1}$ & 6.177 & 6.180 & 6.169 & 6.168 & 6.157 & 6.003 & 3.133 & 0.117 & -2.010 & -1.497 & $1.665^{\mathrm{a}}$ & 1.109 & 0.005 \\
\hline$\varepsilon_{2}$ & 11.217 & 10.696 & 10.498 & 9.890 & 8.629 & 6.348 & 6.176 & 6.166 & 6.143 & 3.182 & $1.665^{\mathrm{a}}$ & 4.386 & 6.083 \\
\hline$\varepsilon_{3}$ & 15.466 & 15.104 & 13.514 & 12.593 & 11.440 & 10.930 & 10.472 & 9.594 & 7.088 & 6.174 & 6.163 & 6.170 & 6.284 \\
\hline$\varepsilon_{4}$ & & 19.174 & 18.851 & 16.715 & 15.895 & 14.564 & 13.356 & 11.828 & 10.916 & 10.204 & 8.603 & 8.769 & 10.198 \\
\hline$\varepsilon_{5}$ & & & 22.617 & 22.606 & 19.907 & 18.980 & 17.227 & 15.885 & 14.302 & 12.468 & 11.150 & 10.952 & 11.776 \\
\hline$\varepsilon_{6}$ & & & & 25.739 & 26.237 & 22.768 & 21.839 & 19.750 & 18.164 & 16.126 & 14.544 & 13.556 & 14.597 \\
\hline$\varepsilon_{7}$ & & & & & 28.525 & 29.669 & 25.216 & 24.344 & 21.704 & 20.086 & 17.906 & 16.375 & 17.268 \\
\hline$\varepsilon_{8}$ & & & & & & 30.980 & 32.494 & 27.057 & 26.481 & 22.980 & 21.671 & 19.220 & 19.940 \\
\hline$\varepsilon_{9}$ & & & & & & & 33.226 & 34.241 & 27.965 & $28.072^{\mathrm{a}}$ & 23.706 & 22.572 & 22.760 \\
\hline$\varepsilon_{10}$ & & & & & & & & 35.196 & 35.335 & $28.072^{\mathrm{a}}$ & $28.493^{a}$ & 23.588 & 24.339 \\
\hline$\varepsilon_{11}$ & & & & & & & & & 36.137 & 36.011 & $28.493^{\mathrm{a}}$ & $28.614^{\mathrm{a}}$ & 28.253 \\
\hline$\varepsilon_{12}$ & & & & & & & & & & 36.278 & $36.299^{\mathrm{a}}$ & $28.614^{\mathrm{a}}$ & 28.453 \\
\hline$\varepsilon_{13}$ & & & & & & & & & & & $36.299^{\mathrm{a}}$ & $36.334^{\mathrm{a}}$ & 34.532 \\
\hline$\varepsilon_{14}$ & & & & & & & & & & & & $36.334^{\mathrm{a}}$ & 34.537 \\
\hline
\end{tabular}

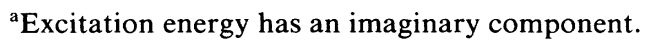

tion. Although the fact that the excitation energies generally do turn out to be real may be used as internal evidence that the approximation scheme is a good one, the appearance of complex eigenvalues for this system is not unexpected for large values of the coupling constant. For example, when considering the random-phase approximation for small oscillations about the unperturbed ground state (i.e., our normal model state), Lipkin et al. ${ }^{1}$ showed that for a value of $g$ within the deformed phase, the collective excited state has an imaginary energy eigenvalue. This was taken as an indication that the unperturbed ground state is unstable against these vibrations, and that another solution to the Hartree-Fock equations exists, ${ }^{2}$ namely, just the state $\left|\Phi^{\prime}\right\rangle$ of Sec. III. It is clear that the appearance of complex eigenvalues in our approximate ECCM treatment is a manifestation of the same problem. For large values of $g$, the excited states are simply too different from the starting model state to be easily obtained by our method at a low level of truncation. Nevertheless, with the exception of the very closely spaced low-lying and nearly degenerate parity doublets, our method still does surprisingly well in this region. An examination of the critical regime, $1 \leq g \leq 2$, in Fig. 2, shows no sharp transition but rather a gradual deterioration of the approximate solutions as $g$ increases. The results for higher values of $N$ are also quite similar.

Perhaps the most interesting feature of these results in the deformed region is their convergence properties. A typical example is illustrated in Fig. 3 for the $N=30$ system at a value $g=5$ for the coupling parameter. For this case the ground and first excited states are almost completely degenerate. Even the sixth and seventh excited states only differ in energy by about $0.5 \%$. At reasonably low levels of truncation in the excited-state ECCM hierarchy of equations, the results that we obtain for the excitation energies are a very good approximation to the average values of each of the parity doublets, but the level splitting or the near degeneracy is entirely absent. As the excited-state truncation index $m$ is increased, we begin to see the typical phenomenon of level crossing or level repulsion as the approximation becomes more refined, and hence more able to reflect the level splitting. Physically, the reason why our ECCM calculations have difficulty in describing the regime of very small level splitting is clear. Thus the ECCM is basically a local formalism. It deals with a set of linked-cluster amplitudes that for a real many-body system are quasilocal in character, insofar as they all obey the cluster property. Conversely, parity is a global quantity and, as such, is not

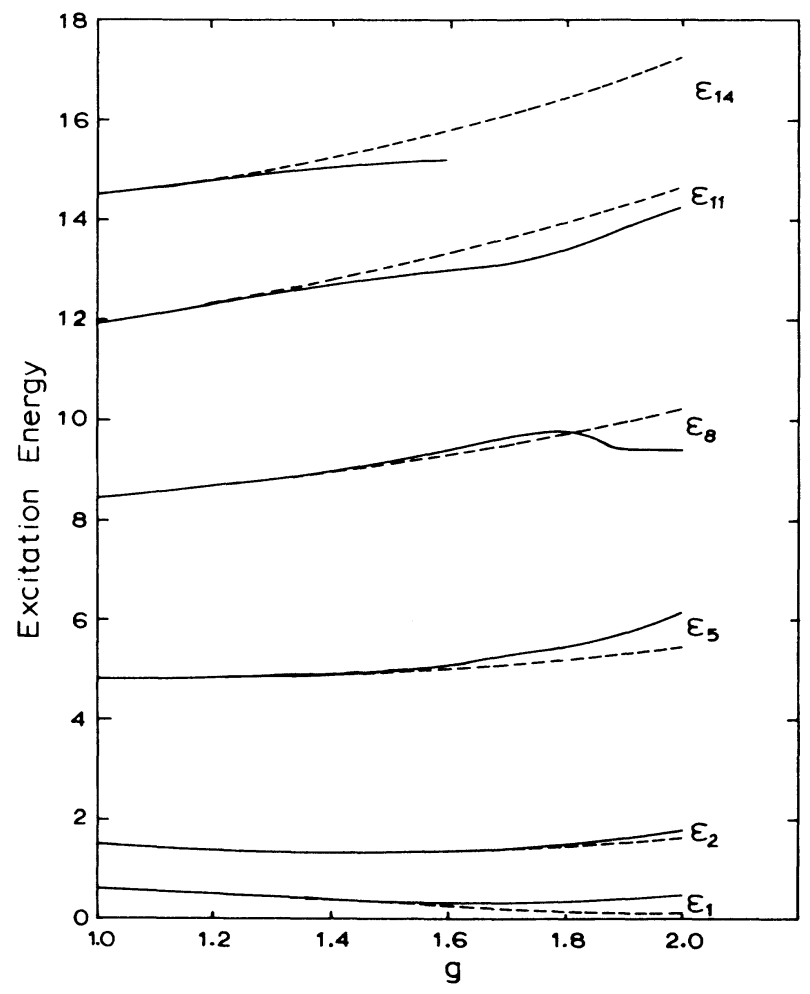

FIG. 2. A comparison of the exact and approximate values of several excitation energies of the $N=14$ system in the critical regime $1 \leq g \leq 2$. The solid lines are the results of our ECCM calculation in a SUB(14,6) approximation based on the normal model state, and the dashed line are exact results. 


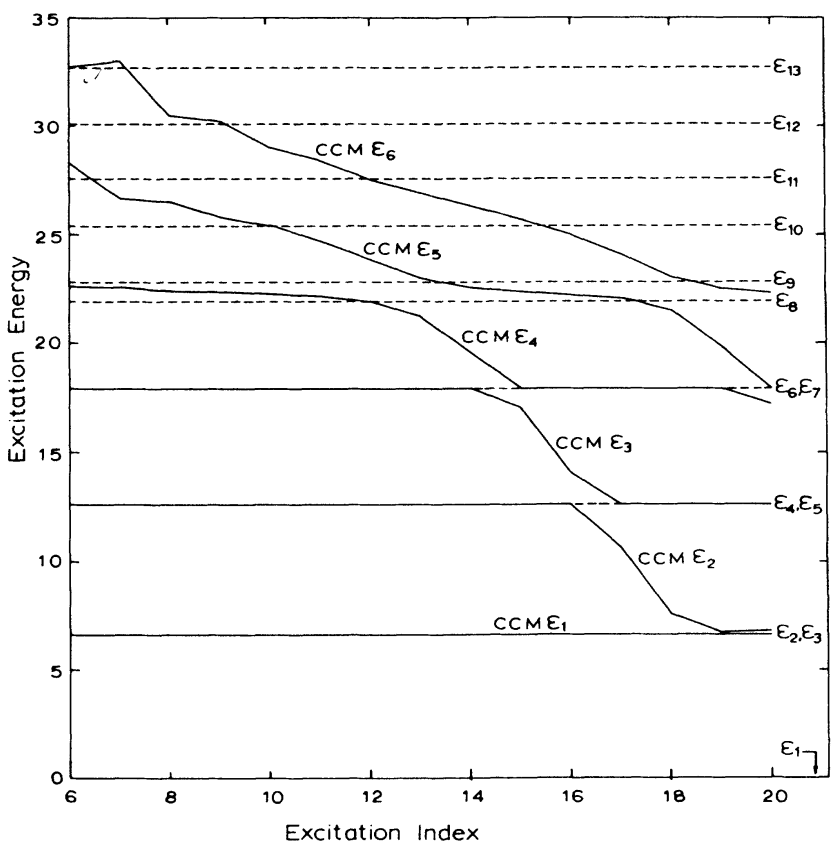

FIG. 3. Convergence properties of the lowest six ECCM excitation energies $\varepsilon_{k}$ (solid lines) as a function of the excitation index $m$ in a $\operatorname{SUB}(m, 6)$ truncated calculation based on the normal model state, for an $N=30$ system with coupling parameter $g=5$. The horizontal dashed lines are the corresponding exact results. (Note that the lowest eight states form four nearly degenerate parity doublets, none of which can be resolved on the scale displayed.)

easily able to be represented in terms of a set of quasilocal parameters. Another way of saying the same thing is that the tunneling through the potential barrier which causes the degeneracy to be lifted for the parity doublets, is also a global phenomenon. It is necessary to sample a large number of (virtual) excited states, particularly at least some of these with energies higher than the height of the barrier, before we can expect to see any evidence of the tunneling.

Figure 3 also demonstrates that the convergence is not uniform as the excited-state truncation index $m$ is increased. As each approximate ECCM excitation energy approaches an exact value it becomes temporarily pinned to it. These "plateaus of convergence" are a characteristic of all of our deformed-phase results. It is clear that the results of these calculations have not yet fully converged. The truncation index $m$ could certainly be increased further. However, the presence of the complex eigenvalues in the excitation energy spectrum suggests that this is not likely to be profitable without increasing the accuracy of the ground-state input, as we have seen that there is no absolute guarantee of convergence to the exact results even if the excitation index $m \rightarrow N$ for a fixed value $n<N$.

Before proceeding to compare the above results with those obtained using the deformed Hartree-Fock state as model wave function, it is instructive to return briefly to the question of multiple ground-state solutions in the
$\operatorname{SUB}(n)$ approximation, to which we have already alluded. Typically we find that the deformed solutions (i.e., with nonzero odd-indexed amplitudes $\left.S_{i}, S_{i}^{\prime \prime}\right)$ at a given $\operatorname{SUB}(n)$ level of approximation exist only above some critical value $g_{c}$, which itself depends on the truncation index $n$. The corresponding symmetric solution, on the other hand, usually continues to exist as $g$ is smoothly increased from zero to values even appreciably above the respective critical value $g_{c}$. Such solutions presumably become increasingly unstable against small perturbations until again some upper critical point is reached beyond which they no longer exist. We may observe from Fig. 4 the typical crossing behavior of the two corresponding estimates for the ground-state energy as a function of the coupling constant $g$. Thus out of the two approximate solutions based on the same normal (symmetric) model state, namely, the parity-preserving and the paritybroken approximations, the symmetry-breaking solution does not give the best approximation to the ground-state energy for values of $g$ all the way down to the corresponding value $g_{c}$. There is actually a narrow range of values above $g_{c}$ in which the symmetry-preserving approximation has an energy closer to the exact result.

Figure 4 also displays the striking general feature that all of our ground-state ECCM calculations based on the symmetric (normal) Hartree-Fock solution as model state, in both the symmetric and deformed phases, give energies which are always upper bounds to the corresponding exact results. It is not known at present whether this variational feature is accidental or is indicative of a more general result. One will appreciate that it clearly does not follow trivially from our discussion in Sec. II, since our whole formulation is not manifestly Hermitian. Since the energy $E_{g}$ has thus not been calculated as a strict expectation value between states which are Hermitian adjoints of each other, the usual proof of the variational theorem certainly does not apply.

\section{B. The deformed (symmetry-breaking) model state}

We have already seen in Sec. III that the normal model state does not minimize the Hartree-Fock energy in the corresponding approximation to the deformed phase, but locally maximizes it. We might expect, therefore, to obtain better ECCM results at a given $\operatorname{SUB}(n)$ level of truncation in the deformed phase by using the deformed Hartree-Fock state $\left|\Phi^{\prime}\right\rangle$ as model state. The state $\left|\Phi^{\prime}\right\rangle$ is thus given by Eqs. (3.16) and (3.17) or, equivalently, by Eq. (3.22), with the angle $\alpha$ given by the symmetrybreaking Hartree-Fock value $\alpha=\cos ^{-1}\left[g^{-1} N /(N-1)\right]$ of Eq. (3.25). In this case the ground-state energy of Eq. (4.8) is now minimized in the $\operatorname{SUB}(n)$ approximation with respect to the parameters $\left\{S_{i}, S_{i}^{\prime \prime} ; i=1, \ldots, n\right\}$, with the coefficients $h_{n}$ replaced by the corresponding $h_{n}^{\prime}$ given by Eq. (4.18). Typical results are again displayed in Fig. 4, where we show two separate branches of SUB(4) solutions based on the deformed model state. It is clear that the branch which exists for all values of $g \geq N /(N-1)$, the critical value in Hartree-Fock approximation, now leads to values of the ground-state energy $E_{g}$ which no longer have the upper-bound property except for a very 


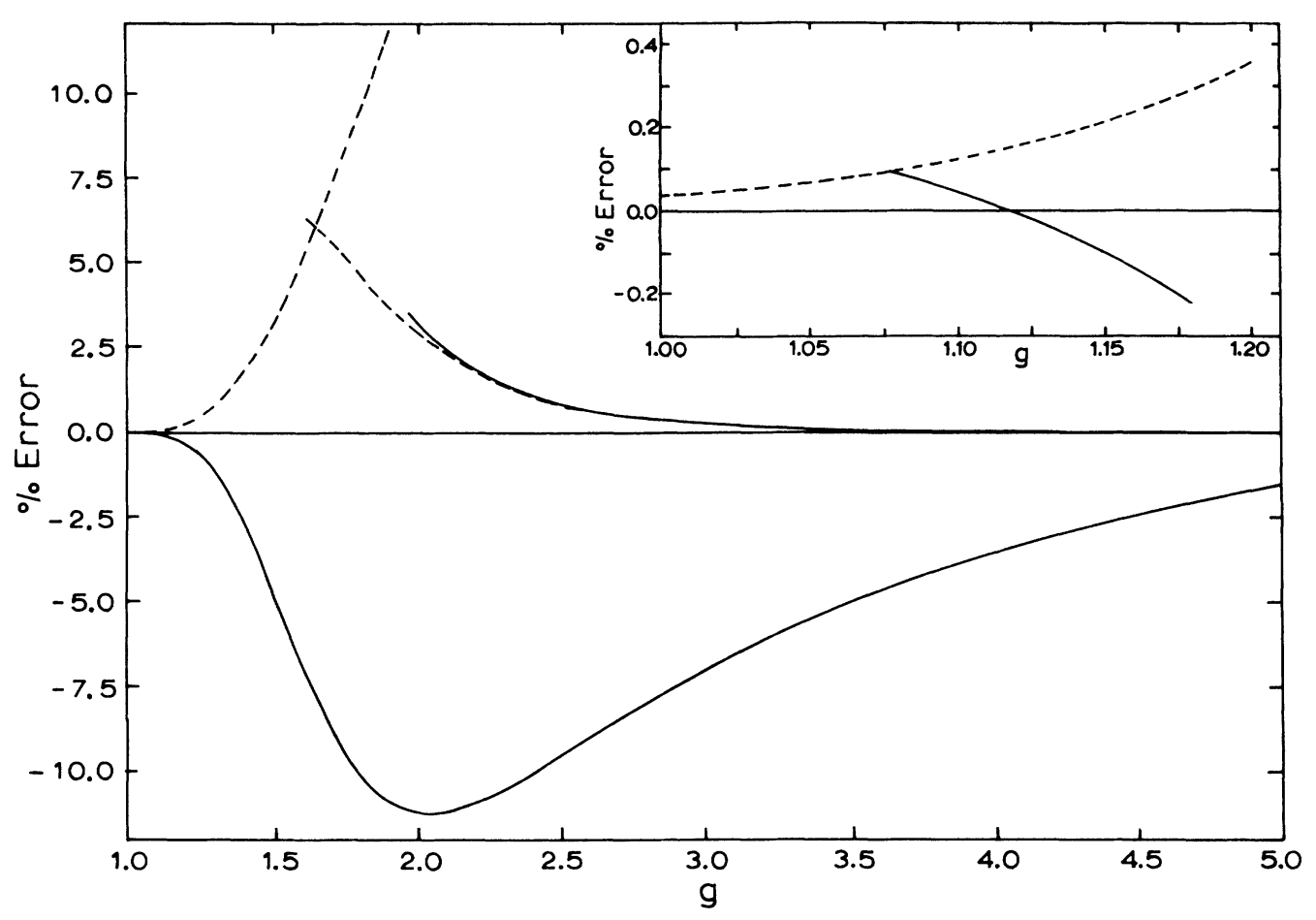

FIG. 4. The error $\left(1-E_{0} / E_{0}^{\text {exact }}\right)$, expressed as a percentage, in the ground-state correlation energy $E_{0} \equiv-E_{g}-N / 2$, for the $N=14$ system, as a function of the coupling parameter $g$, and with $E_{g}$ calculated from four different ECCM solutions obtained in the SUB(4) approximation. The dashed lines represent two different solutions based on the normal model state $|\Phi\rangle$, while the solid lines represent two different solutions based on the deformed Hartree-Fock model state $\left|\Phi^{\prime}\right\rangle$.

narrow range of values of $g$ near the Hartree-Fock critical value. On the other hand, a second branch is also seen to exist above some new critical value of $g$. The corresponding approximate value of the ground-state energy has the properties that: (i) it is always above the exact result, and hence has the same seemingly variational feature as the approximate ECCM solutions based on the normal model state, (ii) it is generally much more accurate than the solution which does not have the upper bound property, and (iii) it is generally very close to the corresponding approximate ECCM value based on the normal model state. In this last respect it is particularly striking that the energy obtained from this particular branch of solutions based on the deformed model state only becomes more accurate than that based on the normal model state for values of $g$ greater than some particular value which is well into the deformed region, e.g., for $g \gtrsim 3.0$ in the case $N=14$ displayed in Fig. 4 .

Especially for these larger values of the coupling constant the results are extremely accurate. This is particularly true for large values of $N$ also, as may be seen from Table VI. This general trend is to be expected since the Hartree-Fock approximation itself becomes asymptotically exact as the limits $N \rightarrow \infty, g \rightarrow \infty$ are approached. Nevertheless, the detailed accuracy attainable over a very broad range of the parameters $N$ and $g$ is impressive. It is also very interesting to note from Fig. 4 how remarkably close in this case are the two estimates for the ground- state energy from the two branches of solutions shown which lie higher than the exact energy in the deformed phase, despite being built on the very different model wave functions $|\Phi\rangle$ and $\left|\Phi^{\prime}\right\rangle$.

Despite this similarity in the above two estimates for the ground-state energy, the excitation energies that are calculated from the corresponding two SUB(4) approximations to the ground-state wave functions differ appreciably. Results from the previous case employing the normal model-state wave function $|\Phi\rangle$ have been shown at a value $g=5$ for the coupling constant in Table V. The corresponding results using the deformed Hartree-Fock model state $\left|\Phi^{\prime}\right\rangle$ are demonstrated for the same $N=14$ system in Table VII and Fig. 5. We note immediately that although the qualitative pattern of the convergence and other comparable properties of the results are similar, the quantitative agreement with exact results is now very much improved. Furthermore, complex values of the ECCM excitation energies appear much less frequently, and occur only for the highest excited states, which are very nearly doubly degenerate.

Another particularly noticeable feature of these solutions based on the deformed Hartree-Fock model state is that the convergence of our ECCM estimates for the excitation energies to individual levels, as opposed to the nearly degenerate parity doublets, is appreciably slower than for the corresponding solution based on the normal model state, as the excited-state truncation index $m$ is in- 
TABLE VI. The ground-state correlation energy, $E_{0} \equiv-E_{g}-\frac{1}{2} N$, in various $\operatorname{SUB}(n)$ approximations for a particular branch of solutions based on the deformed (symmetry-breaking) Hartree-Fock model state which preserves the upper-bound property, compared with exact results.

\begin{tabular}{|c|c|c|c|c|c|c|c|c|c|c|}
\hline \multirow[b]{2}{*}{$N$} & \multirow[b]{2}{*}{ Method } & \multicolumn{9}{|c|}{$g$} \\
\hline & & 1.2 & 1.4 & 1.6 & 1.8 & 2.0 & 2.5 & 3.0 & 4.0 & 5.0 \\
\hline \multirow[t]{4}{*}{14} & SUB(2) & $\mathrm{a}$ & 0.592901 & 0.748019 & 1.145191 & 1.574661 & 2.817984 & 4.199826 & 7.165001 & 10.262375 \\
\hline & SUB(4) & $\mathrm{a}$ & $\mathrm{a}$ & $\mathrm{a}$ & $\mathrm{a}$ & 1.581632 & 2.819291 & 4.200357 & 7.166459 & 10.265955 \\
\hline & SUB(6) & $\mathrm{a}$ & a & $\mathrm{a}$ & a & $\mathrm{a}$ & 2.820063 & 4.200684 & 7.166594 & 10.266040 \\
\hline & Exact & 0.418346 & 0.621287 & 0.890984 & 1.231581 & 1.636084 & 2.843360 & 4.212242 & 7.170901 & 10.268351 \\
\hline \multirow[t]{4}{*}{30} & SUB $(2)$ & $\mathrm{a}$ & 0.900021 & 1.628891 & 2.528115 & 3.542297 & 6.393551 & 9.512373 & 16.148026 & 23.047850 \\
\hline & SUB(4) & a & a & 1.636945 & 2.532958 & 3.545729 & 6.395519 & 9.513762 & 16.148925 & 23.048535 \\
\hline & SUB(6) & a & a & 1.637779 & 2.533132 & 3.545806 & 6.395543 & 9.513775 & 16.148931 & 23.048539 \\
\hline & Exact & 0.543178 & 0.966355 & 1.652829 & 2.537457 & 3.547115 & 6.395653 & 9.513792 & 16.148932 & 23.048539 \\
\hline \multirow[t]{4}{*}{50} & SUB(2) & 0.591433 & 1.455379 & 2.743313 & 4.297513 & 6.035117 & 10.887807 & 16.173881 & 27.393183 & 39.042880 \\
\hline & SUB(4) & 0.656501 & 1.462457 & 2.746867 & 4.299826 & 6.036811 & 10.888812 & 16.174598 & 27.393649 & 39.043233 \\
\hline & SUB(6) & a & 1.462865 & 2.746922 & 4.299847 & 6.036822 & 10.888815 & 16.174600 & 27.393650 & 39.043234 \\
\hline & Exact & 0.666326 & 1.471912 & 2.747668 & 4.299915 & 6.036830 & 10.888815 & 16.174600 & 27.393650 & 39.043234 \\
\hline
\end{tabular}

No solution exists on this branch of $\operatorname{SUB}(n)$ results for this value of $g$.

creased. For example, the deformed model-state results shown for the $N=30$ system in Fig. 6 may be compared with the analogous earlier results based on the normal model state shown in Fig. 3. This slower convergence is a direct consequence of the fact that the starting point of our ECCM expansion now corresponds to the bottom of either one of the two degenerate double wells, rather than the central maximum midway between them which is the comparable starting point in the case of the normal model state in the deformed region. As a consequence, one has to sample excited states even further away from the starting point before the presence of the other well is felt and, hence, before we can accurately describe the level splitting caused by the tunneling between them.
Finally, we return to the alternative approximate ground-state solutions built upon the deformed HartreeFock model state and consider the excited states constructed from them as input. Thus, for example, Fig. 4 demonstrates that for the $N=14$ system with a value $g=2.0$ for the coupling constant, we may compare results for the excitation energies from at least three separate SUB(4) inputs-namely, the one based on the normal model state which allows both even- and odd-indexed coefficients $\left\{S_{n}, S_{n}^{\prime \prime}\right\}$, and the two distinct branches based on the deformed model state. Results are shown in Table VIII for each of these ground-state inputs, in the SUB $(14,4)$ approximation in each case.

A close inspection clearly reveals that the set of results

TABLE VII. The excitation energies $\varepsilon_{n}$ for the $N=14$ system with $g=5.0$, in various $\mathrm{SUB}(m, n)$ approximations based on the deformed (symmetry-breaking) model state and labeled by the truncation indices $(m, n)$, compared with exact results. The ground-state CCM input is based on the upper branch of deformed SUB(4) solutions which preserve the upper-bound property, and which is shown in Fig. 4 as the upper solid curve.

\begin{tabular}{|c|c|c|c|c|c|c|c|c|c|c|c|c|c|}
\hline & $(3,4)$ & $(4,4)$ & $(5,4)$ & $(6,4)$ & $(7,4)$ & $(8,4)$ & $(9,4)$ & $(10,4)$ & $(11,4)$ & $(12,4)$ & $(13,4)$ & $(14,4)$ & Exact \\
\hline$\varepsilon_{1}$ & 6.199 & 6.190 & 6.186 & 6.183 & 6.181 & 6.179 & 6.178 & 6.176 & 6.171 & 5.552 & 3.158 & 0.000 & 0.005 \\
\hline$\varepsilon_{2}$ & 11.363 & 11.013 & 10.835 & 10.669 & 10.500 & 10.262 & 9.831 & 8.952 & 7.508 & 6.195 & 6.180 & 6.104 & 6.083 \\
\hline$\varepsilon_{3}$ & 16.496 & 15.842 & 14.855 & 14.182 & 13.440 & 12.670 & 11.872 & 11.248 & 10.859 & 10.534 & 9.749 & 6.265 & 6.284 \\
\hline$\varepsilon_{4}$ & & 20.776 & 20.099 & 18.606 & 17.715 & 16.734 & 15.878 & 15.018 & 14.114 & 12.968 & 11.420 & 10.200 & 10.198 \\
\hline$\varepsilon_{5}$ & & & 24.493 & 24.001 & 22.083 & 21.228 & 20.001 & 18.944 & 17.707 & 16.330 & 14.506 & 11.735 & 11.776 \\
\hline$\varepsilon_{6}$ & & & & 27.580 & 27.317 & 24.835 & 24.258 & 22.667 & 21.527 & 19.665 & 17.243 & 14.564 & 14.597 \\
\hline$\varepsilon_{7}$ & & & & & 30.041 & 29.939 & 26.569 & 26.484 & 23.934 & 22.573 & 19.803 & 17.257 & 17.268 \\
\hline$\varepsilon_{8}$ & & & & & & 31.923 & 31.888 & 27.317 & 27.304 & 23.955 & 22.677 & 19.944 & 19.940 \\
\hline$\varepsilon_{9}$ & & & & & & & 33.264 & 33.247 & 28.130 & 28.037 & 24.347 & 22.798 & 22.760 \\
\hline$\varepsilon_{10}$ & & & & & & & & 34.061 & 34.054 & 28.456 & 28.315 & 24.350 & 24.339 \\
\hline$\varepsilon_{11}$ & & & & & & & & & 34.384 & 34.391 & 28.482 & 28.321 & 28.253 \\
\hline$\varepsilon_{12}$ & & & & & & & & & & 34.455 & $34.466^{\mathrm{a}}$ & 28.513 & 28.453 \\
\hline$\varepsilon_{13}$ & & & & & & & & & & & $34.466^{\mathrm{a}}$ & $34.469^{\mathrm{a}}$ & 34.532 \\
\hline$\varepsilon_{14}$ & & & & & & & & & & & & $34.469^{\mathrm{a}}$ & 34.537 \\
\hline
\end{tabular}

${ }^{a}$ Excitation energy has an imaginary component. 


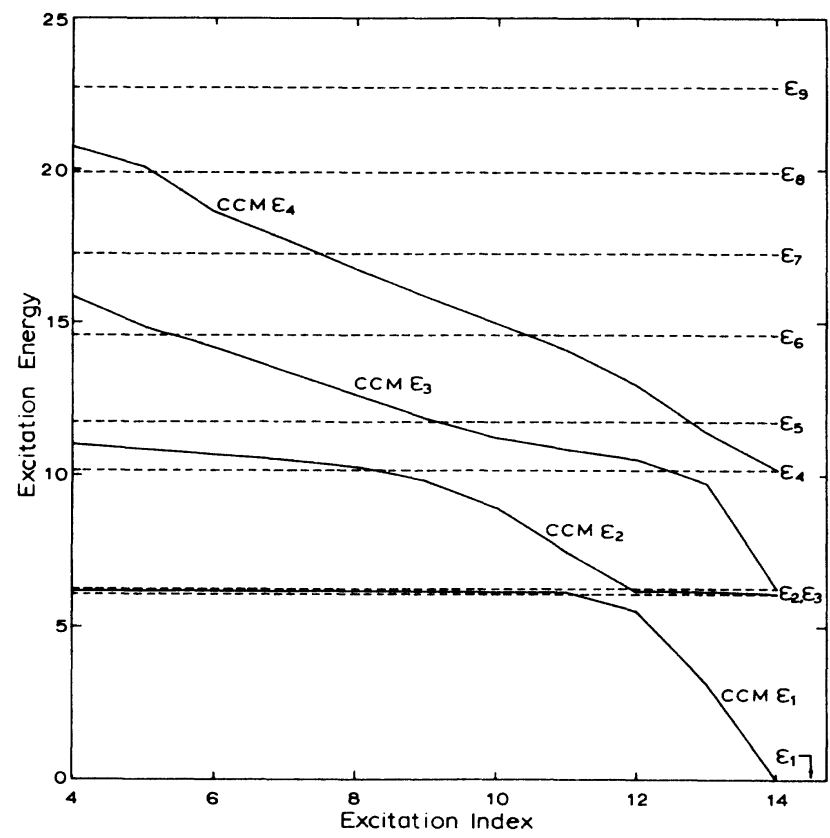

FIG. 5. Convergence properties of the lowest four ECCM excitation energies $\varepsilon_{k}$ (solid lines) as a function of the excitation index $m$ in a $\operatorname{SUB}(m, 4)$ truncated calculation based on the deformed Hartree-Fock model state, for an $N=14$ system with coupling parameter $g=5$. The ground-state SUB(4) input corresponds to the upper solid curve of Fig. 4. The horizontal dashed lines are the corresponding exact results.

based on the upper branch of solutions utilizing the deformed Hartree-Fock model state shown in Fig. 4, is considerably more accurate than either of the other two sets. In this case complex pairs of eigenvalues only occur for the highest two excited states, and then only in

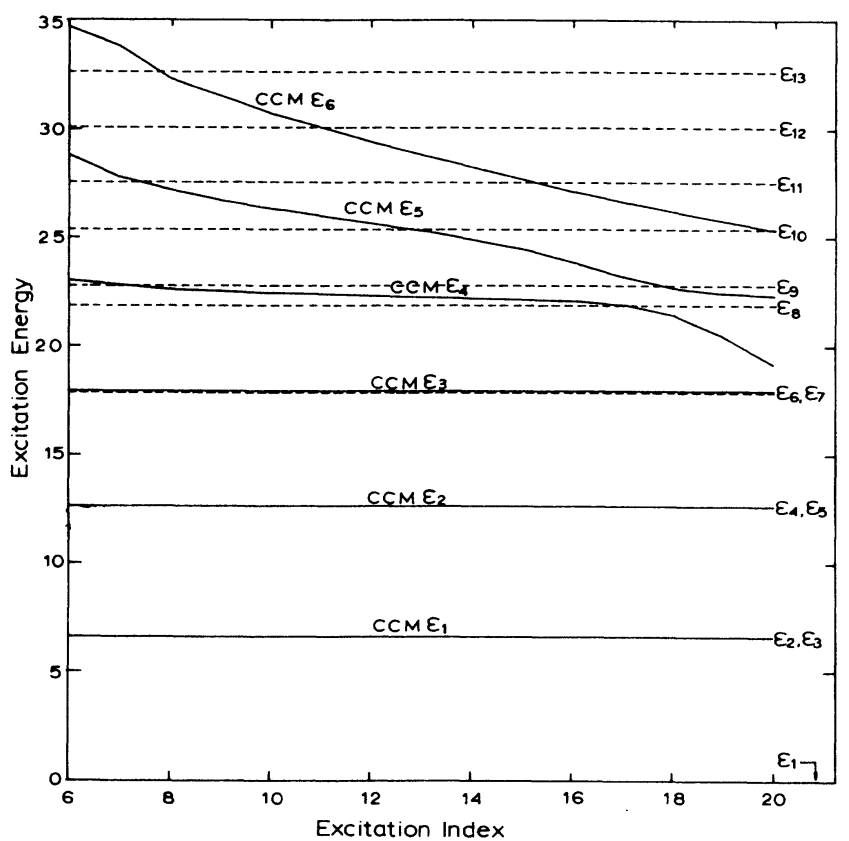

FIG. 6. As Fig. 3, but with the ECCM solutions based on the corresponding deformed Hartree-Fock model state.

$\operatorname{SUB}(m, 4)$ approximations with $m \geq 12$. In each case the imaginary part is very small furthermore. Typically it never exceeds in magnitude about $1 \%$ of the corresponding real part.

By contrast the excited-state results based on the normal model state with comparable SUB(4) ground-state input are not as accurate, even though this approximation gives a marginally more accurate value for the groundstate energy. In this case complex eigenvalues also only

TABLE VIII. The ground-state correlation energy $E_{0} \equiv-E_{g}-\frac{1}{2} N$, and the excitation energies $\varepsilon_{n}$ for the $N=14$ system with $g=2.0$, at the SUB $(14,4)$ level of approximation based on various model states, compared with exact results. The column labeled $N$ indicates results based on the normal model state with both even- and odd-indexed CCM amplitudes $\left\{S_{i}, S_{i}^{\prime \prime}\right\}$, whereas the columns labeled $D_{1}$ and $D_{2}$ indicate results from the upper and lower branches, respectively, of ground-state solutions based on the deformed Hartree-Fock model state, as shown in Fig. 4.

\begin{tabular}{llrrr}
\hline \hline & $N$ & $D_{1}$ & $D_{2}$ & Exact \\
\hline$E_{0}$ & 1.588 & 1.582 & 1.820 & 1.636 \\
$\varepsilon_{1}$ & 0.479 & -0.043 & 0.183 & 0.119 \\
$\varepsilon_{2}$ & 1.758 & 1.464 & 1.202 & 1.653 \\
$\varepsilon_{3}$ & 2.921 & 2.456 & 2.216 & 2.611 \\
$\varepsilon_{4}$ & 4.388 & 3.907 & 3.809 & 3.997 \\
$\varepsilon_{5}$ & 5.947 & 5.482 & 5.774 & 5.472 \\
$\varepsilon_{6}$ & 7.509 & 7.136 & 7.825 & 7.035 \\
$\varepsilon_{7}$ & 8.415 & 8.804 & 9.937 & 8.636 \\
$\varepsilon_{8}$ & 9.479 & 10.434 & $12.502+1.291 i$ & 10.237 \\
$\varepsilon_{9}$ & 11.093 & 11.995 & $12.502-1.291 i$ & 11.800 \\
$\varepsilon_{10}$ & 12.698 & 13.353 & 12.863 & 13.275 \\
$\varepsilon_{11}$ & 14.199 & 14.630 & $15.273+1.024 i$ & 14.661 \\
$\varepsilon_{12}$ & 15.361 & 15.282 & $15.273-1.024 i$ & 15.619 \\
$\varepsilon_{13}$ & $16.994+0.581 i$ & $16.954+0.128 i$ & $16.987+5.983 i$ & 17.153 \\
$\varepsilon_{14}$ & $16.994-0.581 i$ & $16.954-0.128 i$ & $16.987-5.983 i$ & 17.272 \\
\hline \hline
\end{tabular}


occur in $\operatorname{SUB}(m, 4)$ approximations for the excitation energies with $m \geq 12$, but the complex eigenvalues are no longer only restricted to the highest excited states. The imaginary parts are also larger, although in magnitude they still do not exceed about $7 \%$ of the corresponding real parts.

Finally, both of the above sets of results may be contrasted with the comparable ones based on the lower branch of the ground-state solutions shown in Fig. 4 which utilize the deformed Hartree-Fock model state. In this case complex eigenvalues occur in $\operatorname{SUB}(m, 4)$ approximations with values of $m$ as low as four. The complex eigenvalues are neither restricted to the upper portion of the spectrum nor are their imaginary parts small. In general they are comparable in magnitude to the real parts. Furthermore, the overall pattern of convergence as the excited-state truncation index $m$ is increased, is extremely erratic in this case. For example, the lowest two excitation-energy eigenvalues, which are given in the SUB $(14,4)$ approximation by the reasonably accurate values of 0.18 and 1.20 , are given in the $\operatorname{SUB}(6,4)$ approximation as $-8.81 \pm 3.84 i$, in the $\operatorname{SUB}(7,4)$ approximation as -23.81 and -5.86 , and in the $\operatorname{SUB}(8,4)$ approximation as $-8.21 \pm 15.17 i$.

\section{SUMMARY AND DISCUSSION}

The coupled-cluster method, particularly in its extended (ECCM) form, provides us with one of the most universal and widely applicable of all formulations of quantum many-body theory. In its dynamica formulation it is capable, at least in principle, of describing both equilibrium and nonequilibrium behavior, and such intrinsically nonperturbative aspects of many-body systems as spontaneous symmetry breaking, phase transitions, and topological excitations and deformations. Earlier papers in this series ${ }^{52,54}$ have shown how the formalism may be regarded as an exact extension and generalization of both mean-field theory and random-phase approximation (RPA), and how the ECCM amplitudes which fully and exactly parametrize the given many-body system obey a (generalized) classical Hamiltonian mechanics. In particular we have explicitly shown ${ }^{54}$ how the ECCM may be used to construct an effective second-order Hamiltonian approach to small oscillations, by linearizing the equations of motion around a stationary equilibrium point. This exact generalized RPA leads to a set of smalloscillation (linear response) collective eigenmodes, which were further shown to correspond precisely to the (collective) excited states parametrized within the ECCM as in Eqs. (2.14)-(2.17) here.

In the present paper an alternative prescription for finding the excitation spectrum within the ECCM has been formulated within the static formalism in terms of a variational principle for a new excitation-energy functional. This new version is reminiscent of Emrich's prescription for excited states within the older NCCM, with which it shares the advantage of being able to choose independently the levels of approximation for the descriptions of the ground and excited states. In this new method a given ECCM approximation for the ground state, calculated at the $\operatorname{SUB}(n)$ level of truncation, may be used as input to the excited-state formulation at the $\operatorname{SUB}(m, n)$ level of truncation in order to calculate $m$ excited states, where $m$ and $n$ are independent indices. Conversely, in the most natural approximate implementation of the dynamic linear response formulation, a given $\operatorname{SUB}(n)$ approximation for the stationary equilibrium ground state yields an approximation to the same number $n$ of excited states.

Previously, Arponen and Rantakivi ${ }^{9}$ have very successfully applied the dynamic ECCM method of small oscillations to the LMG quasispin model, in order to demonstrate the general power of the entire ECCM formulation. The present method has now also been similarly applied to the LMG model, and in particular the extra flexibility has been exploited to calculate many more excited states. We have again demonstrated that the method is capable of spanning the region of the shape (or phase) transition, using a single (unperturbed or uncorrelated) model state, to give an excellent description of the energy eigenstates in both the symmetric and deformed phases. Furthermore, we have also examined the sensitivity of the method to the choice of the model state, by using both the symmetric and symmetry-breaking Hartree-Fock solutions, which are always fully equivalent to the ECCM SUB(1) solutions, whether symmetry breaking does or does not occur.

The general overall quality of the results is extremely encouraging even at relatively low levels of truncation, outside a narrow critical region whose width decreases with increasing particle number $N$. Indeed, we know of no equally fundamental microscopic formalism that can give better overall results for the LMG model. In the deformed phase the accuracy of our method is improved by choosing the deformed Hartree-Fock solution as the model state. We have seen that in the regime of high coupling constant $g$ where the parity doublets are very nearly degenerate in energy, the excited-state truncation index has to be pushed to rather large values in order to resolve with even rough accuracy, their splitting due to quantum tunneling. On the other hand, the convergence in this regime to the degenerate (unsplit) values is again very rapid. The consequent overall convergence properties in this highly deformed region are well illustrated by the "plateaus of convergence" behavior seen in Figs. 3 and 6 .

In summary, we see that the present ECCM formulation, which is based on a single model or reference state, can rather readily and accurately reproduce virtually all of the qualitative and quantitative details of the energy spectrum. The one noticeable exception is the splitting of the nearly degenerate parity doublets that arise in the deformed region due to the symmetry breaking. It is therefore of interest both to consider the reasons for this, and how they might be remedied. We have already alluded earlier to the underlying reason for the difficulty in reproducing the level splitting. It resides in the basic dichotomy between the very important and fundamental feature for the ECCM of providing a complete description of the many-body system in terms of a set of linked-cluster (and hence basically local) amplitudes, and the essentially global nature of the symmetry-breaking phenomenon. 
Finally, we enquire how we might attempt to refine the ECCM further, in order to handle such phenomena more directly, rather than by a brute-force increase in the truncation indices, which although possible for the LMG model considered here is not likely to be practicable for more realistic systems. In the first place, one might imagine a different truncation scheme so that the effects of the broken symmetry are built in at each level in some suitable fashion. A practical scheme of such a nature is not easy to devise, however. Furthermore, it would also be much more satisfying from a physical viewpoint to have the effect built into the formalism from the outset by a suitable modification to the model or uncorrelated state.

In this respect, we have already seen that of the Hartree-Fock solutions of Eqs. (3.16) and (3.17), neither the symmetric nor the symmetry-breaking case given by the respective parameter choices in Eq. (3.25) is wholly satisfactory for a discussion of level splitting. In order to improve upon these choices, we have two options. First, we could perform a full maximum-overlap calculation ${ }^{6}$ in order to optimize fully the model state at a given level of approximation. In fact, this simply reduces to the same form of model state as in Eqs. (3.16) and (3.17), but with a different choice of the angle parameter $\alpha$. Alternatively, we could relax the present restriction to a single model state and turn instead to a multireference state approach. It seems fairly evident that what is probably needed is to restore the broken symmetry by projecting out the states of good parity. We would thus first form the usual even and odd linear combinations of the two degenerate model states with equal and opposite particular values for the parameter $\alpha$, as done by Agassi et al. ${ }^{2}$ in their deformed Hartree-Fock calculations, and then try to construct a new ECCM approach based on these two model states considered together on an equal footing from the outset.

We have performed some further maximum-overlap calculations, for the LMG model, of the sort described above. The main outcome is that different choices of the parameter $\alpha$, away from the Hartree-Fock values, do not markedly improve the description of level splitting within the ECCM. This is hardly surprising in view of the previous discussion, since it seems clear that we need from the start some way of incorporating the global symmetry property. In other words, we need to give the two degenerate, deformed model states (for a given choice of parameter $\alpha$ ) equal emphasis, in order to restore the symmetry.

Precisely the same point has arisen earlier in Ref. 43 , in the context of a NCCM treatment of double-well anharmonic oscillators. For a fuller discussion of many general points which are also pertinent here, we refer the interested reader to the concluding section of that paper. Briefly, although various extensions of the NCCM to a multireference state approach have been given, ${ }^{17,24,35,62,63}$ the existing versions have essentially been designed with open-shell systems in mind. Their inclusion of multiple reference states thus models the small (and essentially local) changes in the occupations of a set of quasidegenerate valence orbitals, rather than the incorporation of a pair of degenerate, deformed model states, as considered here, that differ only by a global discrete symmetry property. These standard multireference state approaches are, therefore, likely to be of little use in the brokensymmetry case considered here. Indeed, the general broken-symmetry case is more fundamental than the cases addressable by the existing multireference methods, since even the single-particle orbitals must now be taken differently in the different (multireference) Hartree-Fock states. There is no doubt that such an extension of the ECCM, or indeed of the NCCM, as envisaged above, would find applications not only to the present LMG model, but also to many other physical systems where spontaneous symmetry breaking is an important feature.

\section{ACKNOWLEDGMENTS}

One of us (R.F.B.) gratefully acknowledges support for this work in the form of a research grant from the Science and Engineering Research Council of Great Britain.
${ }^{1}$ H. J. Lipkin, N. Meshkov, and A. J. Glick, Nucl. Phys. 62, 188 (1965); 62, 199 (1965); 62, 211 (1965).

${ }^{2}$ D. Agassi, H. J. Lipkin, and N. Meshkov, Nucl. Phys. 86, 321 (1966).

${ }^{3}$ A. Faessler and A. Plastino, Z. Phys. 220, 88 (1969).

${ }^{4}$ R. M. Dreizler and A. Klein, Phys. Rev. C 7, 512 (1973).

${ }^{5}$ R. Shankar and K. K. Gupta, Z. Phys. A 280, 73 (1977).

${ }^{6}$ H. G. Kümmel, Nucl. Phys. A317, 199 (1979).

${ }^{7}$ K. Emrich, Nucl. Phys. A351, 397 (1981).

${ }^{8}$ A. Klein and C. T. Li, Phys. Rev. Lett. 46, 895 (1981).

${ }^{9}$ J. Arponen and J. Rantakivi, Nucl. Phys. A407, 141 (1983).

${ }^{10}$ A. P. Zuker, M. Dufour, and C. Pomar, in Condensed Matter Theories, edited by J. S. Arponen, R. F. Bishop, and M. Manninen (Plenum, New York, 1988), Vol. 3, p. 29.

${ }^{11}$ F. Coester, Nucl. Phys. 7, 421 (1958).

${ }^{12}$ F. Coester and H. G. Kümmel, Nucl. Phys. 17, 477 (1960).

${ }^{13}$ R. F. Bishop and H. G. Kümmel, Phys. Today 40 (3), 52 (1987).
${ }^{14}$ H. G. Kümmel, Nucl. Phys. A176, 205 (1971).

${ }^{15}$ H. G. Kümmel and K. H. Lührmann, Nucl. Phys. A191, 525 (1972).

${ }^{16}$ K. H. Lührmann and H. G. Kümmel, Nucl. Phys. A194, 225 (1972).

${ }^{17}$ R. Offermann, W. Ey, and H. G. Kümmel, Nucl. Phys. A273, 349 (1976); R. Offermann, ibid. A273, 368 (1976).

${ }^{18}$ H. G. Kümmel, K. H. Lührmann, and J. G. Zabolitzky, Phys. Rep. 36C, 1 (1978).

${ }^{19}$ H. G. Kümmel, in Nucleon-Nucleon Interaction and Nuclear Many-Body Problems, edited by S. S. Wu and T. T. S. Kuo (World Scientific, Singapore, 1984), p. 46.

${ }^{20}$ J. Čižek, J. Chem. Phys. 45, 4256 (1966).

${ }^{21}$ J. Paldus, J. Čižek, and I Shavitt, Phys. Rev. A 5, 50 (1972).

${ }^{22}$ D. Mukherjee, R. K. Moitra, and A. Mukhopadhyay, Pramana (India) 4, 247 (1975).

${ }^{23}$ H. J. Monkhorst, Int. J. Quantum Chem. Symp. 11, 421 (1977). 
${ }^{24}$ I. Lindgren, Int. J. Quantum Chem. Symp. 12, 33 (1978).

${ }^{25}$ R. J. Bartlett and G. D. Purvis, Int. J. Quantum Chem. 14, 561 (1978).

${ }^{26}$ V. Kvasnička, V. Laurinc, and S. Biskupič, Phys. Rep. 90C, 160 (1982).

${ }^{27}$ K. Szalewicz, J. G. Zabolitzky, B. Jeziorski, and H. J. Monkhorst, J. Chem. Phys. 81, 2723 (1984).

${ }^{28}$ W. D. Laidig, G. D. Purvis, and R. J. Bartlett, J. Phys. Chem. 89, 2161 (1985).

${ }^{29}$ H. Nakatsuji, J. Chem. Phys. 83, 713 (1985).

${ }^{30}$ H. Sekino and R. J. Bartlett, J. Chem. Phys. 84, 2726 (1986).

${ }^{31}$ M. Takahashi and J. Paldus, J. Chem. Phys. 85, 1486 (1986).

${ }^{32}$ J. Noga and R. J. Bartlett, J. Chem. Phys. 86, 7041 (1987).

${ }^{33}$ U. Kaldor, J. Chem. Phys. 87, 467 (1987).

${ }^{34}$ S. Pal, M. Rittby, R. J. Bartlett, D. Sinha, and D. Mukherjee, Chem. Phys. Lett. 137, 273 (1987).

${ }^{35}$ I. Lindgren and D. Mukherjee, Phys. Rep. 151, 93 (1987).

${ }^{36}$ R. F. Bishop and K. H. Lührmann, Phys. Rev. B 17, 3757 (1978).

${ }^{37}$ R. F. Bishop and K. H. Lührmann, Phys. Rev. B 26, 5523 (1982).

38 J. Arponen and E. Pajanne, J. Phys. C 15, 2665 (1982); 15, 2683 (1982).

${ }^{39}$ K. Emrich and J. G. Zabolitzky, Phys. Rev. B 30, 2049 (1984).

${ }^{40}$ C. S. Hsue and J. L. Chern, Phys. Rev. D 29, 643 (1984).

${ }^{41}$ U. B. Kaulfuss and M. Altenbokum, Phys. Rev. D 33, 3658 (1986).

${ }^{42}$ H. G. Kümmel, in Ref. 10, p. 21.

${ }^{43}$ R. F. Bishop and M. F. Flynn, Phys. Rev. A 38, 2211 (1988).

${ }^{44}$ R. F. Bishop, M. C. Boscá, and M. F. Flynn, Phys. Lett. 132A, 440 (1988).

${ }^{45}$ M. Altenbokum and H. G. Kümmel, Phys. Rev. D 32, 2014
(1985).

${ }^{46}$ U. Kaulfuss, Phys. Rev. D 32, 1421 (1985).

${ }^{47}$ U. Kaulfuss and M. Altenbokum, Phys. Rev. D 35, 604 (1987).

${ }^{48}$ M. Funke, U. Kaulfuss, and H. G. Kümmel, Phys. Rev. D 35 , 621 (1987).

${ }^{49} \mathrm{H}$. Primas, in Modern Quantum Chemistry, edited by $\mathrm{O}$. Sinanoglu (Academic, New York, 1965), Vol. II, p. 45.

${ }^{50}$ R. K. Nesbet, Phys. Rev. 109, 1632 (1958).

${ }^{51}$ J. Arponen, Ann. Phys. (N.Y.) 151, 311 (1983).

52J. S. Arponen, R. F. Bishop, and E. Pajanne, Phys. Rev. A 36, 2519 (1987).

${ }^{53}$ D. J. Thouless, The Quantum Mechanics of Many-Body Systems (Academic, New York, 1961).

${ }^{54}$ J. S. Arponen, R. F. Bishop, and E. Pajanne, Phys. Rev. A 36, 2539 (1987).

${ }^{55}$ D. Bohm and D. Pines, Phys. Rev. 82, 625 (1951); 92, 609 (1953); D. Pines, ibid. 92, 626 (1953).

56 J. S. Arponen, R. F. Bishop, E. Pajanne, and N. I. Robinson, Phys. Rev. A 37, 1065 (1988).

${ }^{57}$ J. Arponen, R. F. Bishop, and E. Pajanne, in Condensed Matter Theories, edited by P. Vashishta, R. K. Kalia, and R. F. Bishop (Plenum, New York, 1987), Vol. 2, p. 373.

${ }^{58}$ K. Emrich, Nucl. Phys. A351, 379 (1981).

${ }^{59}$ J. Goldstone, Proc. R. Soc. London, Ser. A 239, 267 (1957).

${ }^{60}$ R. Chowdhuri, D. Mukherjee, and M. D. Prasad, in Aspects of Many-Body Effects in Molecules and Extended Systems, Vol. 50 of Lecture Notes in Chemistry, edited by D. Mukherjee (Springer, Berlin, 1989), p. 3.

${ }^{61}$ R. F. Bishop and A. Vourdas, J. Phys. G 11, 95 (1985).

${ }^{62}$ B. Jeziorski and H. J. Monkhorst, Phys. Rev. A 24, 1668 (1981).

${ }^{63}$ D. Mukherjee, in Ref. 10, p. 67. 